\title{
Random matching and assignment under dichotomous preferences
}

\author{
Anna Bogomolnaia Hervé Moulin \\ Southern Methodist University Rice University
}

October 2001 


\begin{abstract}
We consider bilateral matching problems where each person views those on the other side of the market as either acceptable or unacceptable: an acceptable mate is preferred to remaining single, and the latter to an unacceptable mate; all acceptable mates are welfare-wise identical.

Using randomization, many efficient and fair matching methods define strategyproof revelation mechanisms. Randomly selecting a priority ordering of the participants gives a simple example.

Equalizing as much as possible the probability of getting an acceptable mate accross all participants stands out for its normative and incentives properties: the profile of probabilities is Lorenz dominant, and the revelation mechanism is groupstrategyproof for each side of the market.

Our results apply to the random assignment problem as well.

Keywords: matching, assignment, strategyproofness, dichotomous preferences
\end{abstract}

Anna Bogomolnaia, Department of Economics, Southern Methodist University (SMU), P.O. Box 0496, Dallas, TX 75275-0496, USA, annab@mail.smu.edu,

Hervé Moulin, Department of Economics, MS 22, Rice University, P.O. Box 1892, Houston, TX 77251-1892, USA, moulin @ rice.edu, http://www.ruf.rice.edu/ econ/papers/index.html

\title{
JEL: C78, C71, D63
}

Moulin's research is supported by the NSF under grant SES0112032 .

We are grateful to seminar and conference participants in the Université de Paris, Seoul National University, the State University of New York at Stony Brook, and Michigan State University. 


\section{Random matching and assignment under dichotomous preferences}

\section{Introduction}

The bilateral matching problem occupies a special place in the mechanism design literature, combining strong empirical relevance and an interesting mathematical structure (see Roth and Sotomayor [1990]). The celebrated Gale-Shapley algorithm selects an efficient matching with strong incentive properties: it is stable in the sense of the core, and strategyproof with respect to the side of the market actively proposing to the other side (though not with respect to the passive side).

We consider the important special case of the bilateral matching problem where each man (resp. woman) evaluates each woman (resp. man) as acceptable or unacceptable: being matched with an acceptable (resp. unacceptable) mate is better (resp. worse) than remaining single, and two acceptable mates yield the same welfare. Abusing language slightly, we speak of dichotomous preferences, to capture the idea that the preferences of an individual are entirely described by the subset of his or her acceptable mates ${ }^{1}$.

For convenience we use the marriage terminology, but we have in mind other kinds of matching than sharing a life - for which a single binary criterion is an utterly insufficient model! Examples relevant to our model include matching managers to support staff, when a staff person is acceptable to a manager if and only if he has certain skills, and a manager is acceptable to a staff person if and only if she is not requesting "hard" tasks; or matching professors to research assistants, when the professor is acceptable to the student if and only if he works in a certain area, and the student is acceptable to the professor if and only if he is willing to do certain chores; or matching pilots to copilots, nurses to doctors, and so on. For this kind of matching problems, time-sharing is a simple and commonly observed tool to divide fairly the indivisible market participants. Randomizing over different matchings is formally equivalent to time-sharing, and is the accepted terminology in the assignment problem discussed below. We adopt it for matching as well.

In the classical bilateral matching model, the assumption that preferences over potential mates are strict is essential: see sections 2.3 and 2.5.1 in Roth and Sotomayor [1990]. By contrast, widespread indifferences are the very subject of our model, and indeed our results differ sharply from those of the classical model. The main new feature is that the problem of efficiently matching a set $M$ of men and a set $W$ of women, all with dichotomous preferences, decomposes in two disjoint subproblems $M^{o}, W^{d}$ and $M^{d}, W^{o}$. The women in $W^{d}$ compete

\footnotetext{
${ }^{1}$ Yet individual's preferences have at least three indifferences classes corresponding to being matched with an acceptable mate, or an unacceptable one, or remaining single. Moreover, preferences among unacceptable mates do not matter, as we only consider voluntary (individually rational) matchings.
} 
for the men in $M^{o}$, and the latter can - and will, in any efficient matching - all be matched to some subset of $W^{d}$. The roles of men and women are reversed in $M^{d}, W^{o}$. Because the only action in the efficient subproblem $M^{o}, W^{d}$ is which women get matched and which don't, we can think of $M^{o}$ as a set of passive "objects", to be assigned to the agents in $W^{d}$. In this view, the matching problem reduces to a pair of assignment problems, a crucial simplification allowing us to develop in parallel the analysis of the matching and the assignment problems.

Dichotomous preferences are equally natural in the assignment problems. Think of housemates distributing single rooms, when a "good" room may be one with a private bath for some agent, one with a private phone for another agent, and so on; another example is the assignment of softwares to workers when a given software can be compatible or not with a worker's own machine; or the scheduling of a list of jobs by a single server among a given set of timeslots: each customer requesting one job finds only certain time-slots acceptable (e.g., the job is useless after next Tuesday, or can only be done on week-ends, and so on).

Barring monetary compensations, we use randomization (time-sharing) to achieve a fair compromise among efficient matchings, or assignments. This normative interpretation of randomization is not new in the literature on assignment (see Section 2), but it does not appear in the matching literature. Our main finding is that the three enduring goals of mechanism design, efficiency, fairness and incentive compatibility, are compatible under dichotomous preferences.

In the rest of this section, we outline our results. The first observation is that, in the dichotomous domain, the set of matchings stable in the sense of the core is nothing more than the set of efficient and voluntary matchings. Indeed, an objection by an unmatched man and an unmatched woman who are mutually acceptable is also a Pareto improvement ${ }^{2}$. Secondly, the decomposition of efficient and voluntary (deterministic) matchings in two disjoint efficient assignments implies that the total number of men - and women - matched in any efficient matching is a constant $e$, a number that we call the efficiency size of the given matching problem. Conversely, a matching where $e$ men and $e$ women are matched is efficient.

Randomization (time-sharing) is easy to evaluate in the dichotomous domain, because utility takes only two values, 0 if single, and 1 if matched. Thus the probability to get an acceptable mate is the canonical utility function ${ }^{3}$ over random matchings. A crucial consequence of the definition of $e$ follows: a random voluntary matching is efficient ex ante (i.e., with respect to the profile of probabilities of getting an acceptable mate) if and only if its efficient ex post, namely if with probability $1, e$ men and $e$ women are matched.

This fact allows us to construct many efficient, fair and strategyproof matching methods. The simplest example uses the familiar idea of selecting randomly, and with uniform probability, a priority ordering of the participants. We call

\footnotetext{
${ }^{2}$ Note that "weakly improving" objections often lead to an empty core, for instance when we have one woman and two men, and every mate is acceptable to every one.

${ }^{3}$ First order stochastic dominance is a complete preordering of all random matchings, and the axiomatic apparatus of the von Neumann-Morgenstern utility theory is superfluous.
} 
it the random priority solution. For any fixed ordering of the set of men and women, the corresponding lexicographic maximization of utilities over voluntary matchings yields an efficient deterministic matching (unique utility-wise): this matching method is clearly strategyproof. When we randomize with fixed probabilities over all orderings of the set of agents, strategyproofness is clearly preserved, and so is efficiency. The former statement does not depend upon the assumption of dichotomous preferences, the latter one does.

In the spirit of random priority, we construct a different solution averaging over all efficient deterministic utility profiles, and show (Lemma 8) that this uniform solution also combines efficiency, fairness and strategyproofness.

If random priority is an important and versatile idea for fair division, an even more basic concept is that of equalizing utilities in the sense of the leximin ordering, i.e., maximizing first the smallest individual utility, then the next to last utility, and so on. Our main result is that the maximization of the leximin ordering when utilities are the probabilities of being matched delivers a random matching method that stands out both for its normative (Theorem 1) and incentive properties (Theorem 2). The former result says that the utility profile corresponding to this egalitarian solution maximizes much more than the leximin preordering: it first-order stochastically dominates any other feasible profile (when utilities are rearranged increasingly), a property known as Lorenz dominance. This implies for instance that the egalitarian solution maximizes the Nash product of individual utilities, and, more generally, the sum of any concave transformation of individual utilities. Theorem 1 also provides a simple algorithm to compute the egalitarian solution.

The latter result (Theorem 2) establishes the very strong incentive properties of the egalitarian solution related to joint misreport of their preferences by coalitions (subgroups) of agents. Because the domain of dichotomous preferences is of much smaller size than that of strict preferences $\left(2^{n}\right.$ versus $\left.n !\right)$ it is easier for the agents to learn about their mutual preferences, which facilitates the formation of coalitions.

A mechanism is called groupstrategyproof if no coalition can increase the utility of at least one member, without decreasing that of at least one other member. We show that the egalitarian solution is groupstrategyproof with respect to all-male and all-female coalitions (Corollary to Theorem 2).

By contrast, neither the random priority nor the uniform solution are groupstrategyproof with respect to all-male, or all-female, coalitions. Moreover Lemma 7 shows that no deterministic matching method is groupstrategyproof with respect to all-male, or all-female, coalitions. It is easy to check the latter claim for a fixed priority method. Say that three men share two women by the fixed priority: $m_{1} \succ m_{2} \succ m_{3}$. Each woman finds all men acceptable, $m_{1}$ likes $w_{1}$ and $w_{2}, m_{2}$ likes only $w_{1}$, and $m_{3}$ likes only $w_{2}$. For these true preferences $\left(m_{1}, w_{2}\right)$ and $\left(m_{2}, w_{1}\right)$ are matched. However $m_{1}$ can help $m_{3}$ at no cost to himself, by reporting that he likes only $w_{1}$, resulting in the matches $\left(m_{1}, w_{1}\right)$ and $\left(m_{3}, w_{2}\right)$.

When we allow for manipulations by coalitions mixing some men and some women, strong impossibility results obtain. No efficient solution treating equals equally is even weakly groupstrategyproof; the same is true of any solution 
selecting a utility profile without specifying the probabilistic matching to implement it: Theorem 3 .

The paper is organized as follows. Section 2 reviews the relevant literature. The model is defined in Section 3. Efficient voluntary matchings (and assignments), deterministic as well as random, are characterized in Section 4. The egalitarian, random priority and uniform methods are defined and compared in Section 5. Strategyproofness and groupstrategyproofness are the subject of Section 6. Further fairness properties in the random assignment problem are discussed in Section 7; in particular we give a competitive interpretation of the egalitarian random assignment (corollary to Theorem 1). Section 8 gathers some concluding comments.

\section{Relation to the literature}

A bipartite graph between a set of men and a set of women connects some men to some women by edges. A matching is a subset of edges of the graph such that each point (man or woman) is in at most one edge. Matching theory explores the properties of inclusion maximal matchings, simply called maximal matchings. If we interpret the presence (resp. absence) of an edge in the graph joining $m$ to $w$ as "man $m$ and woman $w$ are mutually acceptable", then maximal matchings are precisely efficient and voluntary matchings. The decomposition of efficient matchings in two disjoint efficient assignments is then a simple reinterpretation of the well known Gallai-Edmonds decomposition of bipartite matching graphs: see Theorem 3.2.4 in Lovasz and Plummer [1986], an excellent survey of matching theory.

The economic theory of bilateral matching under strict preferences - surveyed in the classic book by Roth and Sotomayor [1990] — does not address fairness by randomization, but examines in great details strategyproofness and core stability. Roth [1982] shows that no mechanism selecting a core stable matching at all profiles can be strategyproof for both men and women. This incompatibility disappears under dichotomous preferences, because core stability boils down to efficiency and voluntariness: for instance a fixed priority matching method is core stable in the latter domain, but not in the strict preference domain. Theorem 2 and Lemma 8 offer a large family of core stable and strategyproof random matching methods.

In the classical (strict preferences) domain, the man-optimal Gale Shapley algorithm selects the best core stable matching from the point of view of men. This mechanism is groupstrategyproof for the men but manipulable by women (Dubins and Freedman [1981], Gale and Sotomayor [1985]). Our egalitarian solution, by contrast, is groupstrategyproof for either side of the market.

The small literature on random assignment under strict preferences is very relevant to our work. Hylland and Zeckhauser [1979] defined a fair and efficient solution, adapting to the random assignment problem the familiar competitive equilibrium with equal incomes. Yet this competitive solution is not incentive compatible, and in fact Zhou [1990] establishes the general impossibility 
of achieving ex ante efficiency (when preferences over lotteries are described by von Neumann-Morgenstern utilities), fairness (in the minimal sense of equal treatment of equals), and strategyproofness.

If the efficiency requirement is weakened to ex post efficiency (each deterministic assignment chosen with positive probability is Pareto optimal), these three properties are met by the random priority mechanism, ordering the agents randomly and letting them pick successively their best object still available, a well defined procedure under strict preferences. This mechanism is ex post efficient, strategyproof, and treats equals equally. It is not, however, ex ante efficient (Bogomolnaia and Moulin [2000]). Interestingly, it can be interpreted as the competitive equilibrium with random property rights (Abdulkadiroglu and Sönmez [1998]).

Our egalitarian and random priority solutions adapt the above two methods to dichotomous preferences, and extend them to bilateral matching.

A recent flurry of papers on the deterministic assignment of indivisible goods bears some relation to our work. The central question of that literature is to characterize the set of efficient and incentive compatible (strategyproof) assignment mechanisms. The fixed priority mechanisms and the competitive trade mechanisms (from a fixed distribution of property rights over objects) stand out in this set, but interesting compromises emerge as well: Papai [2000]. This literature almost always assumes strict preferences (a few exceptions are discussed below). Fairness in the sense of equal treatment of equals is ruled out because attention is restricted to deterministic assignments. However, the tests of resource and population monotonicity play a role: Ehlers and Klaus [2000], Ehlers, Klaus and Papai [2000].

Svensson [1994], [1999], and Bogomolnaia and Deb [2000] consider the deterministic assignment problem in the full domain of complete and transitive preference relations, thus allowing for strict and for dichotomous preferences. Fixed priority mechanisms are strategyproof and non bossy. Conversely, these two properties force the mechanism to resemble closely a fixed priority one; the additional property of neutrality captures precisely the fixed priority mechanisms. In the same domain of preferences, Wako [1994] shows that competitive trades may not be efficient.

\section{The model}

A matching problem consists of a finite set $M$ of "men", a finite set $W$ of "women", and two $M \times W$ zero-one matrices $R M$ and $R W$, representing dichotomous preferences of men over women and of women over men respectively. An entry $R M_{m w}=1$ if woman $w$ is acceptable for $\operatorname{man} m$, and $R M_{m w}=0$ if she is not acceptable for him (and similarly for $R W$ ). Thus, each row $R M_{m}$ of $R M$ represents the preferences of a man $m$, and each column $R W^{w}$ of $R W$ represents the preferences of a woman $w$.

Each person prefers to be matched to an acceptable person of the opposite gender to being unmatched, but would rather be alone than matched to an 
unacceptable person.

We assume throughout the paper that matching is voluntary, namely two individuals can be matched only if they like each other. We refer to this important assumption as the individual rationality restriction. It implies that all the information about feasible matchings and relevant preferences is conveyed by a single $M \times W$ zero-one matrix $R$, equal to the entry by entry product of $R M$ and $R W: R_{m w}=1$ if and only if $R M_{m w}=1$ and $R W_{m w}=1$, i.e., if and only if man $m$ and woman $w$ are mutually acceptable (we then say that they are "compatible"). We call the triple $(M, W, R)$ the individually rational (ir) reduced problem of the problem $(M, W, R M, R W)$.

Thus we work most of the time with the ir-reduced model $(M, W, R)$. The only exception is Section 6 devoted to strategic behavior: there the matching mechanism computes the ir-reduced problem from the reported preferences $R M$ and $R W$.

As this will cause no confusion, we use the notation $R_{m}$ (resp. $R^{w}$ ) both for the row (resp. the column) of $R$ and for the subset of women (resp. men) who are compatible with man $m$ (resp. woman $w$ ). For any subset $S$ of men (resp. subset $B$ of women) - also called a coalition - we write $R_{S}=\cup_{S} R_{m}$ (resp. $\left.R^{B}=\cup_{B} R^{w}\right)$ for the set of people of the opposite gender, compatible with at least one person in $S$ (resp. $B$ ).

The same triple $(M, W, R)$ can be interpreted in a different way. Suppose we only care about the interests of the one side of the matching market (say, $M)$. We obtain an assignment problem, where we interpret $M$ as the set of (gender neutral) agents, $W$ as the set of objects to be assigned to $M$, and $R$ as a representation of dichotomous preferences of the agents in $M$ over the objects in $W$. We use the same notation as in matching problem, and interpret $R_{m w}=1 / 0$ as "object $w$ is good/bad for agent $m$ ".

When interpreting $(M, W, R)$ as a matching problem, all persons in $M \cup W$ are endowed with preferences, whereas in the assignment interpretation, only the agents in $M$ have preferences ${ }^{4}$.

To distinguish between the matching and assignment interpretations of the problem $(M, W, R)$, we speak of persons in the former, and of agents and objects in the latter. For brevity, we state formal definitions and results in the most convenient interpretation, with a brief mention of the corresponding statement in the alternative interpretation. Moreover, in the case of matching the two sides have perfectly symmetric roles, so we often state a one-sided result (one where the roles of men and women are different) without repeating that the symmetric one-sided result holds as well.

A deterministic matching $\mu$ of the ir-reduced problem $(M, W, R)$ is a subset

\footnotetext{
${ }^{4}$ It turns out that, in the assignment problem, the interpretation of agent's preferences is more general than in matching. In the latter, our results require that "remaining single" be strictly preferred to "being matched to an unacceptable person", whereas in assignment all results are preserved if we only assume that "receiving no object" is not worse than "receiving a bad one"; moreover, receiving no object may not be an option at all. See Comment 2 in Section 8. For the clarity of exposition, we interpret preferences in the assignment and matching problems in the same way.
} 
of $M \times W$, such that $(m, w) \in \mu$ only if $R_{m w}=1$, and any person appears there at most once: for any $m \in M$ (resp. $w \in W$ ) there exist at most one $\widetilde{w} \in W($ resp. $\widetilde{m} \in M)$ such that $(m, \widetilde{w}) \in \mu$ (resp. $(\widetilde{m}, w) \in \mu)$. Persons who appear as a component of a pair from $\mu$, and only those, are matched by $\mu$. We write $\mathcal{A}(M, W, R)$ for the set of deterministic matchings. The definition of a deterministic assignment $\mu$ is identical; we say that an agent $m$ is assigned if and only if there exists an object $\widetilde{w}$, such that $(m, \widetilde{w}) \in \mu$.

A random matching is a lottery $\pi$ on $\mathcal{A}(M, W, R)$. For all our results, the only relevant information about a random matching $\pi$ is the random allocation matrix $Z$, giving for all $m$ and $w$ the probability $z_{m w}$ that man $m$ and woman $w$ are matched, i.e., the probability that $\pi$ selects a deterministic matching $\mu$ such that $(m, w) \in \mu$. Thus the $M \times W$ matrix $Z$ is substochastic, that is to say it is non-negative and the sum of each row and each column is at most 1 ; moreover $z_{m w}$ is positive only if man $m$ and woman $w$ are compatible.

The mapping from a random matching $\pi$ to its allocation matrix $Z$ is clearly not one-to-one. A variant of the Von Neumann-Birkhof theorem on bistochastic matrices (Lemma 2.1 in Bogomolnaia and Moulin [1999]), implies that it is onto the following set of $M \times W$ matrices:

$$
\mathcal{Z}(M, W, R)=\left\{Z \mid Z \text { is substochastic and } R_{m w}=0 \Rightarrow z_{m w}=0\right\}
$$

Given a random allocation matrix $Z$, we interpret the probability that a given person is matched as his or her utility. Denoting by $Z_{m}$ the $m$-th row of $Z$ (man $m$ 's random allocation) and by $Z^{w}$ the $w$-th column of $Z$, we write these utilities as follows:

$$
u_{m}\left(Z_{m}\right)=\sum_{W} z_{m w}=\sum_{R_{m}} z_{m w}, \quad v_{w}\left(Z^{w}\right)=\sum_{M} z_{m w}=\sum_{R^{w}} z_{m w}
$$

We denote by $\mathcal{U} \mathcal{V}(M, W, R)$ the set of feasible utility vectors (of length $|M|+$ $|W|)$, namely the image of $\mathcal{Z}(M, W, R)$ under the utility functions (2). We say that the utility vector in (2) is implemented by $Z$.

When talking about assignment problem, we only look at feasible utility vectors $u$ of length $|M|$ and denote their set by $\mathcal{U}(M, W, R)$.

An (assignment or matching) solution is a mapping $(M, W, R) \rightarrow Z$ associating a random allocation matrix to any problem. A welfarist solution only keeps track of the utility profile: it is a mapping $(M, W, R) \rightarrow(u, v) \in \mathcal{U V}(M, W, R)$ (matching) or $(M, W, R) \rightarrow u \in \mathcal{U}(M, W, R)$ (assignment). The priority solutions defined in Section 4 and the three randomized solutions in Section 5 are welfarist.

\section{Efficiency}

A person who is not compatible with anybody $\left(R_{m}=\emptyset\right.$ or $\left.R^{w}=\emptyset\right)$ has no bearing on efficiency, and can simply be ignored. We assume $R_{m} \neq \emptyset$ and 
$R^{w} \neq \emptyset$ for all $m, w$ in this section and the following one. For future reference, note that such a "null" person could play a strategic role in a group manipulation of the kind we discuss in Section 6 (by sending a non-null report).

For matching problems, if there is a way to simultaneously match all men and all women, we say that $M$ and $W$ can be perfectly matched.

We first show that any efficient matching problem can be decomposed into three disjoint matching problems: one group of men competes for a set of women each of whom always get matched, another set of women competes for another group of always matched men, and the remaining men and women can be perfectly matched.

We introduce this crucial result in an example with ten men and ten women, and the compatibility matrix given in Figure 1. Consider first the subproblem with the set of men $M^{o}=\left\{m_{1}, m_{2}, m_{3}\right\}$ and of women $W^{d}=\left\{w_{6}, w_{7}, w_{8}, w_{9}, w_{10}\right\}$. These three men can be matched to seven (out of ten) different subsets of three women in $W^{d}$ : the three unfeasible subsets are the ones containing both $w_{7}$ and $w_{8}$, whose only mutually acceptable mate is $m_{2}$. Note that any two women in $W^{d}$ who can both be matched (i.e., any pair except $w_{7}, w_{8}$ ) is contained in a triple that can be matched to $M^{\circ}$. It follows that a matching is efficient (and voluntary) in our subproblem if and only if $M^{o}$ is matched to a subset of $W^{d}$, and there are seven such matchings (counting as one all matchings yielding the same utility profile).

Next consider the larger subproblem with additional men $M^{p}=\left\{m_{4}, m_{5}, m_{6}\right\}$ and women $W^{p}=\left\{w_{3}, w_{4}, w_{5}\right\}$. Note that no woman in $W^{d}$ can be matched with any man in $M^{p}$, and that $M^{p}$ and $W^{p}$ have a perfect match. Therefore the efficient matchings in the subproblem $M^{o} \cup M^{p}, W^{d} \cup W^{p}$ simply augment the efficient matchings of $M^{o}, W^{p}$ by the perfect matching of $M^{p}$ to $W^{p}$.

Finally, add the remaining $M^{d}=\left\{m_{7}, m_{8}, m_{9}, m_{10}\right\}$ and $W^{o}=\left\{w_{1}, w_{2}\right\}$. Note that no man in $M^{d}$ can be matched with any woman in $W^{p} \cup W^{d}$, and that $W^{o}$ can be matched with any pair in $M^{d}$. Therefore there are six efficient matchings (utility-wise) of $M^{d}$ and $W^{o}$. We conclude that a matching of the original $10 \times 10$ problem is efficient (and voluntary) if and only if $M^{p}$ and $W^{p}$ are perfectly matched, $M^{o}$ is matched to a feasible triple in $W^{d}$, and $W^{o}$ is matched to a pair in $M^{d}$.

The structure of the efficient and voluntary matchings in the above example is fully general. In the following statement, $\left\{S_{1}, S_{2}, S_{3}\right\}$ is called a partition of $S$ if $S_{1}, S_{2}, S_{3}$ are disjoint subsets of $S$, their union is $S$, and at least one of $S_{1}, S_{2}, S_{3}$ is non-empty.

Lemma 1 (Gallai-Edmonds decomposition)

Given a matching problem $(M, W, R)$ with $R_{m} \neq \emptyset, R^{w} \neq \emptyset$ for all $m, w$, there is a unique pair of partitions $\left\{M^{o}, M^{p}, M^{d}\right\}$ of $M$ and $\left\{W^{o}, W^{p}, W^{d}\right\}$ of $W$ such that:

i) $W^{d}$ is only compatible with $M^{o}$, and $M^{o}$ is overdemanded by $W^{d}$ : $R^{W^{d}}=M^{o}$ and 


$$
\left|R_{S} \cap W^{d}\right|>|S| \quad \text { for all } S \subseteq M^{o}
$$

ii) $M^{p}$ and $W^{p}$ can be perfectly matched

iii) $M^{d}$ is only compatible with $W^{o}$, and $W^{o}$ is overdemanded by $M^{d}$ : $R_{M^{d}}=W^{o}$ and

$$
\left|R^{B} \cap M^{d}\right|>|B| \quad \text { for all } B \subseteq W^{o}
$$

We call $M^{o}, W^{o}$ the sets of overdemanded persons, $M^{p}, W^{p}$ the sets of perfect persons, and $M^{d}, W^{d}$ the sets of disposable persons.

Note in particular, that $\left|M^{o}\right|<\left|W^{d}\right|,\left|M^{p}\right|=\left|W^{p}\right|$ and $\left|M^{d}\right|>\left|W^{o}\right|$. The problem is trivial when the only non-empty sets are $M^{p}$ and $W^{p}$.

The women in $W^{d}$ are compatible only with men in $M^{o}$ and are enough to make everyone in $M^{o}$ happy: we can assign $M^{o}$ to $W^{d}$. In fact (3) implies $\left|R_{S} \cap W^{d} \backslash\{w\}\right| \geq|S|$, hence by Hall's theorem applied to the assignment of overdemanded men, $M^{o}$ can be assigned to $W^{d} \backslash\{w\}$, for any $w$ in $W^{d}$. This justifies our "disposable" terminology for the women in $W^{d}$.

Lemma 2 Notations as in Lemma 1. Define the efficiency size of problem $(M, W, R)$ as

$$
e=\left|M^{o}\right|+\left|W^{o}\right|+\left|M^{p}\right|=\left|M^{o}\right|+\left|W^{o}\right|+\left|W^{p}\right|
$$

A deterministic matching $\mu \in \mathcal{A}(M, W, R)$ is efficient (Pareto optimal w.r.t. the utilities (2)) if and only if its cardinality $|\mu|=e$, i.e., exactly $2 e$ agents (e men and e women) are matched by $\mu$.

In all efficient matchings, $M^{o}$ is matched to a proper subset of $W^{d}, W^{o}$ is matched to a proper subset of $M^{d}$, and $M^{p}$ and $W^{p}$ are perfectly matched.

When we interpret $(M, W, R)$ as an assignment problem, we are only interested in assigning agents from $M$ and do not care whether objects from $W$ get assigned. Lemma 1 becomes: there is a unique pair of partitions $\left\{M^{a}, M^{d}\right\}$ of $M$ and $\left\{W^{o}, W^{n o}\right\}$ of $W$, such that $M^{a}$ can be assigned to $W^{n o}$, and $W^{o}$ is overdemanded by $M^{d}$ (here $M^{a}=M^{o} \cup M^{p}$ and $W^{n o}=W^{p} \cup W^{d}$ ).

Efficient assignments are those which assign exactly $e=\left|M^{a}\right|+\left|W^{o}\right|$ agents: $M^{a}$ must be assigned to $W^{n o}$, and some $S \subseteq M^{d},|S|=\left|W^{o}\right|$, must be assigned to $W^{o}$.

Back to the matching interpretation, the gist of Lemma 2 is that, if $|\mu|<e$, then there exist another, necessarily efficient, matching $\mu^{\prime},\left|\mu^{\prime}\right|=e, \mu^{\prime} \supset \mu$. From the point of view of welfare, all that matters is which pairs of coalitions $(S, B)$ of size $e$, where $S \subset M, B \subset W$, can be matched and which cannot. We call the former pairs efficient and denote their set by $E(M, W, R)$. A person is disposable if and only if there is an efficient coalition $(S, B)$ to which he or she 
does not belong. Note that by, Lemma 2 , there is also an efficient coalition to which he or she belongs (recall our assumption $R_{w}, R_{m} \neq \emptyset$ ).

The only aspect of the efficiency frontier not determined by the partition of Lemma 1 is this: which coalitions from $M^{d}$ can be (simultaneously) matched? and which coalitions from $W^{d}$ can be matched? In the example of Figure 1 the answer is, respectively, every pair of $M^{d}=\left\{m_{7}, m_{8}, m_{9}, m_{10}\right\}$ and seven triples from $W^{d}=\left\{w_{6}, w_{7}, w_{8}, w_{9}, w_{10}\right\}$ (the three triples containing $w_{7}$ and $w_{8}$ are excluded).

The following notation will be useful. Given $(M, W, R)$ and $S \subseteq M, B \subseteq W$, we write $(S, B, \widetilde{R})$ for the matching problem restricted to men in $S$ and women in $B$; thus $\widetilde{R}$ is the $S \times B$ submatrix of $R$.

By Lemmas 1 and 2 , the initial problem decomposes into three disjoint matching problems: $\left(M^{o}, W^{d}, \widetilde{R}\right),\left(M^{p}, W^{p}, \widetilde{R}\right)$, and $\left(M^{d}, W^{o}, \widetilde{R}\right)$. Leave aside the perfect matching of $M^{p}$ to $W^{p}$. Since overdemanded persons always get a match, we can consider $\left(M^{o}, W^{d}, \widetilde{R}\right)$ and $\left(M^{d}, W^{o}, \widetilde{R}\right)$ as assignment problems: in the first one disposable men play the role of "agents", and overdemanded women the role of "objects", while in the second one disposable women are "agents" and overdemanded men are "objects".

The efficiency size $e=e(M, W, R)$ plays a crucial role throughout the paper. We will need the following properties of $e$.

Lemma 3 Notations as in Lemmas 1,2.

$$
\begin{gathered}
m \in M^{o} \cup M^{p} \Leftrightarrow e(M \backslash m, W, \widetilde{R})=e(M, W, R)-1 \\
m \in M^{d} \Leftrightarrow e(M \backslash m, W, \widetilde{R})=e(M, W, R)
\end{gathered}
$$

Lemma 4 Notations as in Lemmas 1,2. The efficiency size e $(M, W, R)$ is submodular in $M$ and in $W$, and supermodular in $M \times W$. In the following inequalities, we write $e(S, B)$ instead of $e(S, B, \widetilde{R})$ :

$$
\begin{aligned}
e(S, W)+e(T, W) & \geq e(S \cup T, W)+e(S \cap T, W), \text { for all } S, T \subseteq M \\
e(M, B)+e(M, C) & \geq e(M, B \cup C)+e(M, B \cap C), \text { for all } B, C \subseteq W \\
e(S, B)+e\left(S^{\prime}, B^{\prime}\right) & \geq e\left(S, B^{\prime}\right)+e\left(S^{\prime}, B\right), \text { for all } S \subseteq S^{\prime} \text { and all } B \subseteq B^{\prime}
\end{aligned}
$$

A characterization, different from Lemma 2, of the set of efficient pairs $E(M, W, R)$, is derived from priority solutions, a special class of welfarist solutions. Given a priority ordering $\succ$ of $M \cup W$, we define the $\succ$ priority utility profile as the $\succ$ lexicographic maximum over the utility set $\mathcal{U V}$. Thus the highest priority person $i_{1}$ gets utility 1 because he or she is compatible with at least one person; the next person in the priority line, $i_{2}$, gets utility 1 if there is a way to match him or her without affecting the utility of person $i_{1}$, otherwise this person's utility is 0 , and so on. Priority solutions are efficient, and our next result states that any efficient matching can be obtained as one of them. Moreover, 
the efficient pair $(S, B)$ matched under a given priority ordering depends only on the relative position of a person among others of the same gender: the utility of a man does not depend on which women precede him and vice versa.

Lemma 5 Notations as in Lemmas 1,2. Given an ordering $\succ_{M}$ of $M$, and an ordering $\succ_{W}$ of $W$, all priority orderings $\succ$ consistent with them both yield the same utility profile $u\left(\succ_{M}\right), v\left(\succ_{W}\right)$, and match the following pair $S(\succ), B(\succ)$ :

$$
\begin{aligned}
m & \in S(\succ) \Leftrightarrow u_{m}=1 \Leftrightarrow e(T \cup\{m\}, W, \widetilde{R})-e(T, W, \widetilde{R})=1, \\
w & \in B(\succ) \Leftrightarrow v_{w}=1 \Leftrightarrow e(M, C \cup\{w\}, \widetilde{R})-e(M, C, \widetilde{R})=1,
\end{aligned}
$$

where $T$ is the set of agents preceding $m$ in $\succ_{M}$, and $C$ is the set of agents preceding $w$ in $\succ_{W}$. Moreover, every efficient pair $(S, B) \in E(M, W, R)$ obtains as $S(\succ), B(\succ)$ for some ordering $\succ$ of $M \cup W$.

Similarly, in the assignment problem $(M, W, R)$, a coalition of agents is efficient if and only if its size is $e(M, W, R)$ and it can be assigned to $W$. And the set of efficient coalitions coincides with the set of coalitions which are assigned by the $\succ_{M}$ priority solution for some ordering $\succ_{M}$ of $M$.

We turn our attention to efficient random matchings. Their description is greatly simplified by the fact that all efficient deterministic matchings have the same size (Lemma 2). Let $\pi$ be a lottery over $\mathcal{A}(M, W, R)$, the set of deterministic matchings. A necessary condition for the Pareto optimality (efficiency) of $\pi$ with respect to the utilities (2) is that its support contains only efficient matchings (this is the familiar ex-post efficiency property). This condition is sufficient as well: by Lemma 2 every efficient deterministic matching $\mu$ maximizes the joint utility $\sum_{M} u_{m}+\sum_{W} v_{w}=2 e(M, W, R)$, and so does every lottery over these matchings.

The final result of this section offers two more characterizations: of efficient random allocation matrices $Z$ thanks to Lemmas 1,2; and of the utility efficiency frontier thanks to Lemmas $3,4,5$.

Lemma 6 Notations as in Lemmas 1,2.

i) A random matching (assignment) is efficient if and only if, with probability one, it matches $2 e(M, W, R)$ persons (assigns e agents).

ii) A random allocation matrix is efficient if and only if the sum of its entries is $e(M, W, R)$.

iii) A random allocation matrix $Z$ for matching problem is efficient if and only if $z_{m w}>0$ only for $(m, w) \in\left(M^{o}, W^{d}\right) \cup\left(M^{p}, W^{p}\right) \cup\left(M^{d}, W^{o}\right)$, and its restrictions to $\left(M^{p}, W^{p}\right)$, to $\left(M^{o}, W^{d}\right)$ and to $\left(M^{d}, W^{o}\right)$ represent, respectively, an efficient matching between $M^{p}$ and $W^{p}$ (i.e., the restricted matrix is bistochastic), and efficient random assignments of $W^{d}$ to $M^{o}$ and of $M^{d}$ to $W^{o}$.

iv) A utility vector $(u, v) \in[0,1]^{M+W}$ is feasible and efficient if and only if it is a solution of the following system: 


$$
\begin{aligned}
& \sum_{M} u_{m}=\sum_{W} v_{w}=e(M, W, R) ; \sum_{S} u_{m} \leq e(S, W, \widetilde{R}) \text { for all } S \subseteq M, \\
& \sum_{B} v_{w} \leq e(M, B, \widetilde{R}) \text { for all } B \subseteq W
\end{aligned}
$$

We denote by $\mathcal{U V}^{e}(M, W, R)$ the set of efficient feasible utility vectors. In the assignment interpretation, the set of efficient and feasible vectors $u \in[0,1]^{M}$ is denoted by $\mathcal{U}^{e}(M, W, R)$.

Lemma 6 extends to efficient random matchings the three-fold decomposition of efficient deterministic matchings discussed before Lemma 3 . The efficient utility frontier $\mathcal{U} \mathcal{V}^{e}(M, W, R)$ is the cartesian product of the three efficiency frontiers $\mathcal{U} \mathcal{V}^{e}\left(M^{o}, W^{d}, \widetilde{R}\right), \mathcal{U} \mathcal{V}^{e}\left(M^{d}, W^{o}, \widetilde{R}\right)$, and $\mathcal{U} \mathcal{V}^{e}\left(M^{p}, W^{p}, \widetilde{R}\right)$. The latter set is trivial (all utilities are 1); the two former sets are described by their projections on $W^{d}$ and $M^{d}$ respectively (because all utilities are 1 in $M^{o}$ and $W^{o}$ ), namely the efficiency frontier $\mathcal{U}^{e}\left(M^{o}, W^{d}, \widetilde{R}\right)$ of the female assignment problem $\left(M^{o}, W^{d}, \widetilde{R}\right)$ and $\mathcal{U}^{e}\left(M^{d}, W^{o}, \widetilde{R}\right)$ of the male assignment problem $\left(M^{d}, W^{o}, \widetilde{R}\right)$.

$>$ From the above discussion, we can canonically extend any efficient assignment solution - selecting for every problem $(M, W, R)$ a utility vector in $\mathcal{U}^{e}(M, W, R)$ - to an efficient matching solution. The canonical extension gives to $M^{d}$ and $W^{d}$ the same utility as they would get, respectively, in the male assignment problem $\left(M^{d}, W^{o}, \widetilde{R}\right)$ and the female assignment problem $\left(M^{o}, W^{d}, \widetilde{R}\right)$, and utility 1 to everyone else. This fact greatly simplifies the notational burden of the next sections.

Property $i v$ ) applied to the (male) assignment problem $(M, W, R)$ characterizes $\mathcal{U}^{e}(M, W, R)$ by the following system:

$$
\sum_{M} u_{m}=e(M, W, R) ; \sum_{S} u_{m} \leq e(S, W, \widetilde{R}) \text { for all } S \subseteq M
$$

This is the very definition of the core of the formal cooperative game $S \rightarrow$ $e(S, W, \widetilde{R})$, where $e$ sets an upper bound on utilities instead of the more familiar lower bound. This game has no useful interpretation in our model. Two familiar "values", the Shapley value and the egalitarian solution, are known to be in the core if the game is submodular (Shapley [1971], Dutta and Ray [1989] ): they correspond to our random priority and egalitarian solutions, respectively.

\section{The egalitarian solution and two averaging so- lutions}

The egalitarian solution picks an efficient matching (or assignment) equalizing the individual utilities as much as possible, i.e., the probabilities of receiving a 
good object. Its utility profile maximizes in $\mathcal{U V}(M, W, R)$ (or in $\mathcal{U}(M, W, R)$ ) the familiar leximin ordering.

Pick a vector $x$ and rearrange its coordinates increasingly: the resulting vector is denoted $x^{*}$. The leximin ordering compares $x$ and $y$ like the lexicographic ordering compares $x^{*}$ and $y^{*}$. Thus $x$ is strictly preferred to $y$ if $y_{1}^{*}<x_{1}^{*}$, or $\left\{y_{1}^{*}=x_{1}^{*}\right.$ and $\left.y_{2}^{*}<x_{2}^{*}\right\}$, or $\left\{y_{i}^{*}=x_{i}^{*}, i=1,2\right.$, and $\left.y_{3}^{*}<x_{3}^{*}\right\}$, and so on. The leximin ordering has a unique maximum over any convex and compact set $\mathcal{U} \mathcal{V}$ $\left(\right.$ or $\mathcal{U}$ ) of utility profiles ${ }^{5}$, and this profile is on the efficiency frontier $\mathcal{U V}^{e}$ (or $\left.\mathcal{U}^{e}\right)$

In the example of Figure 1, women $w_{7}$ and $w_{8}$ are only compatible with $m_{2}$, therefore $\min \left\{v_{7}, v_{8}\right\} \leq 0.5$ for any feasible utility profile $(u, v)$. Men $m_{7}, m_{8}, m_{9}$ and $m_{10}$ have to share $w_{1}$ and $w_{2}$, so $\min \left\{u_{7}, u_{8}, u_{9}, u_{10}\right\} \leq 0.5$ as well. If we reserve $m_{2}, w_{1}$ and $w_{2}$ for these six disposable persons, they each end up with a probability $\frac{1}{2}$ to be matched. The remaining disposable women $w_{6}, w_{9}$ and $w_{10}$ can share men $m_{1}$ and $m_{3}$, so as to get utility $\frac{2}{3}$ each (give $\frac{2}{3}$ of $m_{3}$ to $w_{10}, \frac{1}{3}$ of $m_{3}$ and $\frac{1}{3}$ of $m_{1}$ to $w_{9}$, and $\frac{2}{3}$ of $m_{1}$ to $w_{6}$ ). All other persons get utility 1 . This is the egalitarian solution.

We turn to a general assignment problem $(M, W, R)$. An important property of the egalitarian solution is related to the partial ordering known as Lorenz dominance and defined as follows:

$$
x \text { lor } y \quad \text { iff } \quad\left\{\sum_{t=1}^{k} x_{t}^{*} \geq \sum_{t=1}^{k} y_{t}^{*} \text { for all } k\right\}
$$

If the arbitrary convex set $\mathcal{U}$ of utility profiles contains a Lorenz dominant element $u^{*}$, this profile has a very strong claim to fairness within the efficiency frontier. Indeed, it achieves the maximum over $\mathcal{U}$ of any collective utility function averse to inequality (in the sense of the Pigou-Dalton transfer principle); it is the unique maximum if the collective utility is strictly averse to inequality ${ }^{6}$. Thus $u^{*}$ maximizes not only the leximin ordering but also the Nash collective utility $\sum_{M} \log u_{m}$, and any collective utility $\sum_{M} f\left(u_{m}\right)$ for any increasing and concave function $f$ (if $f$ is strictly concave, $u$ is the unique maximum), and more.

Theorem 1 states that the egalitarian solution of any matching or assignment problem is Lorenz dominant among all feasible utility profiles.

In the statement of Theorem 1 we use the notations $R(T)$ instead of $R_{T}$, $R(T, B)=R(T) \cap B$ and $r(T, B)=|R(T, B)|$ for $T \subseteq M, B \subseteq W$. For any real valued function $h$, the expression $\arg \min _{S} h(T)$ stands for the subset $T$ of $S$ minimizing $h$ over all subsets of $S$; if several subsets of $S$ reach the minimum, $T$ is the largest one in the sense of inclusion: this is well-defined in our case, because, for our choice of the function $h$, if two subsets minimize $h$ on $2^{S}$, so will their union.

\footnotetext{
${ }^{5}$ see, e.g., Lemma 1.1 in Moulin [1988].

${ }^{6}$ That is, collective utility increases from a Pigou-Dalton transfer. See, e.g., Section 2.5 in Moulin [1988].
} 
The last piece of notation concerns the sequence $T_{k}, k=1,2, \ldots$, of disjoint subsets of $M$ constructed in the Theorem. We write $T_{1, \ldots, k}$ for the union of $T_{1}, T_{2}, \ldots, T_{k}$.

Theorem 1 For any assignment problem $(M, W, R)$, the feasible utility set $\mathcal{U}(M, W, R)$ has a Lorenz dominant element denoted $u^{e}$ and called the egalitarian utility profile.

To compute $u^{e}$, we assume $R_{i} \neq \emptyset$ for all $i$ and define a finite increasing sequence of positive numbers $\alpha_{k}, k=1, \ldots, K$, of which all but at most one are strictly below 1 ; and a partition of $M$ by a sequence $T_{k}, k=1, \ldots, K$, of nonempty coalitions:

$$
\begin{aligned}
M_{0} & =M, W_{0}=W ; M_{k}=M \backslash T_{1, \ldots, k}, W_{k}=W \backslash R\left(T_{1, \ldots, k}\right) ; \\
\alpha_{k} & =\min _{M_{k-1}} \frac{r\left(T, W_{k-1}\right)}{|T|} ; \\
T_{k} & =M_{k-1} \quad \text { if } \alpha_{k} \geq 1 ; \quad=\arg \min _{M_{k-1}} \frac{r\left(T, W_{k-1}\right)}{|T|} \quad \text { if } \alpha_{k}<1
\end{aligned}
$$

The sequences $\alpha_{k}, T_{k}$ stop at $K$ where $T_{1, \ldots, k}=M$. The egalitarian utility profile is:

$$
u_{m}^{e}=\alpha_{k} \text { if } m \in T_{k}, \text { for } k=1, \ldots, K-1 ; \quad u_{m}=\min \left\{1, \alpha_{K}\right\} \text { if } m \in T_{K}
$$

In the canonical extension of the egalitarian solution from assignment to matching, the Lorenz domination property is preserved.

The algorithm (9) is related to the Gallai-Edmonds decomposition (Lemma 1) for assignment problems in the following way: if $\alpha_{K}<1$, then $M^{a}=$ $M \backslash M^{d}=\emptyset$; if $\alpha_{K} \geq 1$, then $T_{K}=M_{K-1}=M^{a}$ and $W_{K-1}=W^{n o}=W^{d} \cup W^{p}$. In all cases, no agent in $M^{d}$ is assigned with probability 1 .

The random allocation matrix (or matrices) $Z$ implementing $u^{e}$ are largely determined by the algorithm (9). The main fact, illustrated by Figure 2, is that the objects in $R\left(T_{k}, W_{k-1}\right)=R\left(T_{k}\right) \backslash R\left(T_{1, \ldots, k-1}\right)$, and only those, are assigned to the agents in $T_{k}$. This fact, and the related competitive interpretation of the egalitarian matrices $Z$, are discussed in the section 7 below (section on assignments).

The intuition behind algorithm (9) is straightforward. Consider $T_{1}$ : even if we reserve $R\left(T_{1}\right)$-i.e., all the objects they like - for these agents, at least one agent in $T_{1}$ will not get more than $\alpha_{1}$ because the total utility of $T_{1}$ cannot exceed $r\left(T_{1}, W\right)$. Thus the maximin utility cannot exceed $\alpha_{1}$ :

$$
\min _{m} u_{m} \leq \alpha_{1} \text { for any } u \in \mathcal{U}(M, W, R)
$$

The egalitarian solution assigns $R\left(T_{1}\right)$ to $T_{1}$ (splitting equally $r\left(T_{1}, W\right)$ among $\left.T_{1}\right)$ and repeats the same computation in the reduced problem $\left(M_{1}, W_{1}, \widetilde{R}\right)$ : 
the key fact is that the new maximin utility $\alpha_{2}$ is greater than $\alpha_{1}$, thus the decision to reserve $R\left(T_{1}\right)$ to $T_{1}$ is vindicated.

The main result in Dutta and Ray [1989], is related to - and used in the proof of - our Theorem 1 . Interpret the set $\mathcal{U}^{e}(M, W, R)$ of efficient utility vectors as the core of the submodular cooperative game $S \rightarrow e(S, W, \widetilde{R})$. Theorem 3 in Dutta and Ray [1989] implies that $\mathcal{U}^{e}(M, W, R)$ contains a Lorenz dominant element (the first statement of our Theorem 1 ) and computes it by an algorithm identical to (9), except that $r(T, B)$ is replaced everywhere by $e(T, B)$, the efficiency size of the reduced problem $(T, B, \widetilde{R})$. See the proof of Theorem 1. Note that $r(T, B)$ is computed by direct inspection of the matrix $R$, whereas computing $e(T, B)$ is the harder task of discovering the Gallai-Edmonds decomposition of the subproblem $(T, B, \widetilde{R})$.

We define now two welfarist solutions working by averaging instead of equalizing. As discussed in Section 1, random priority is a familiar idea in the assignment literature.

Definition 1 The random priority assignment solution is the uniform lottery over all $\succ$ priority solutions, one for each ordering $\succ$ of $M$. Its utility profile for the problem $(M, W, R)$ is given by the formula:

$$
u_{m}^{r p}=\sum_{p=0}^{|M|-1} \sum_{T \in \mathcal{S}_{p}(M \backslash m)} \frac{p !(|M|-p-1) !}{|M| !}\{e(T \cup\{m\}, W, \widetilde{R})-e(T, W, \widetilde{R})\}
$$

for all $m ; \mathcal{S}_{p}(M \backslash m)$ is the set of coalitions of size $p$ not containing $m$

In view of Lemma 5, the canonical extension of random priority to matching problems can also be defined as the uniform lottery over all $\succ$ priority matching solutions, one for each ordering $\succ$ of $M \cup W$.

In the example of Figure 1, the random priority utilities for disposable persons are:

$$
u_{7}^{r p}=u_{9}^{r p}=\frac{7}{12} ; \quad u_{8}^{r p}=u_{10}^{r p}=\frac{5}{12} ; \quad v_{7}^{r p}=v_{8}^{r p}=\frac{1}{2} ; \quad v_{6}^{r p}=v_{9}^{r p}=v_{10}^{r p}=\frac{2}{3} ;
$$

Consider man $m_{7}$ : he competes for women $w_{1}$ and $w_{2}$ with $m_{8}, m_{9}$ and $m_{10}$. His utility is 1 if he is the first or the second agent in $\succ_{M^{d}}$ (probability $\frac{1}{4}$ ), or if he is the third and $m_{9}$ is not before him (proba. $\frac{1}{4} \cdot \frac{1}{3}$ ). He is not matched if he is the fourth. Man $m_{8}$ is matched if he is the first (proba. $\frac{1}{4}$ ), or if he is the second and $m_{9}$ is the first (proba. $\frac{1}{4} \cdot \frac{1}{3}$ ). Women $w_{7}$ and $w_{8}$ have to share $m_{2}$, so each of them is matched if and only if she precedes the other one. Finally, women $w_{6}, w_{9}$ and $w_{10}$ can only be matched with $m_{1}$ or $m_{2}$, and each of them is matched whenever she is not the last of the three (proba. $\frac{2}{3}$ ). 
The allocation matrix implementing the above utility profile is not unique in this example. Its restriction to the assignment problem $\left(M^{d}, W^{o}, \widetilde{R}\right)$ could, for example, be

$$
\begin{aligned}
& Z_{7}=Z_{9}=\frac{1}{2} w_{1}+\frac{1}{12} w_{2} ; Z_{8}=Z_{10}=\frac{5}{12} w_{2} ; \\
& \text { or } \quad Z_{7}=\frac{5}{12} w_{1}+\frac{2}{12} w_{2} ; Z_{9}=\frac{7}{12} w_{1} ; Z_{8}=Z_{10}=\frac{5}{12} w_{2} ;
\end{aligned}
$$

Our second averaging solution is similar to-and somewhat more natural than-random priority. For the matching problem $(M, W, R)$, the utility profiles associated with the efficient coalitions are the extreme points of the efficiency frontier $\mathcal{U V}^{e}(M, W, R$ ) (Lemma 6). It is natural to average (by means of a uniform lottery) directly over the set $E(M, W, R)$ of efficient coalitions instead of indirectly over priority orderings: there is no compelling reason to give more weight to an efficient coalition because it is matched under a larger number of priority coalitions. Given the decomposition of $E(M, W, R)$ in Lemma 2, this amounts to average over the efficient coalitions in the two disjoint assignment problems $\left(M^{d}, W^{o}, \widetilde{R}\right)$ and $\left(M^{o}, W^{d}, \widetilde{R}\right)$.

In our example, we have five efficient coalitions for the male assignment problem $\left(M^{d}, W^{o}, \widetilde{R}\right)$ : we can choose any two men out of $M^{d}=\left\{m_{7}, m_{8}, m_{9}, m_{10}\right\}$ except the pair $\left(m_{8}, m_{10}\right)$. Each of $m_{7}$ and $m_{9}$ belongs to three of them, so their utility is $\frac{3}{5}$, while that of $m_{8}$ and $m_{10}$ is $\frac{2}{5}$. For the female assignment problem $\left(M^{o}, W^{d}, \widetilde{R}\right)$, efficient coalitions include one women from the set $\left\{w_{7}, w_{8}\right\}$ and two from $\left\{w_{6}, w_{9}, w_{10}\right\}$, so there are six of them. We obtain that

$$
u_{7}^{u n}=u_{9}^{u n}=\frac{3}{5} ; \quad u_{8}^{u n}=u_{10}^{u n}=\frac{2}{5} ; \quad v_{7}^{u n}=v_{8}^{u n}=\frac{1}{2} ; \quad v_{6}^{u n}=v_{9}^{u n}=v_{10}^{u n}=\frac{1}{3} .
$$

Definition 2 The uniform assignment solution for the problem $(M, W, R)$ is the following utility vector, where $E(M, W, R)$ denotes the set of efficient coalitions in $M$ :

$$
\begin{aligned}
u_{m}^{u n} & =\frac{\left|E_{m}(M, W, R)\right|}{|E(M, W, R)|} \text { for all } m, \\
\text { where } E_{m}(M, W, R) & =\{S \in E(M, W, R) \mid m \in S\}
\end{aligned}
$$

The uniform matching solution is the canonical extension of the above solution to matching. In view of Lemma 2, it can also be defined by the above formula, where $E(M, W, R)$ is the set of efficient coalitions $S, B$ of the matching problem.

\section{Strategyproofness and groupstrategyproofness}

We investigate in this section the strategic opportunities in the direct revelation mechanisms associated with solutions (or welfarist solutions) of the as- 
signment or matching problems. Recall that a solution $g$ maps every problem $(M, W, R)$ into a random allocation matrix $Z$, whereas a welfarist solution $f$ maps $(M, W, R)$ into a utility profile $u$ (or $(u, v))$. Thus every solution $g$ projects onto the following welfarist solution $f(M, W, R)=u(g(M, W, R))$.

Strategyproofness is not a welfarist concept: its definition requires to specify how the allocation matrix is affected when the reported preferences change. When we speak below of a welfarist solution (such as the three solutions defined in the previous section) being strategyproof (or any group variant of this property) we always refer to the most demanding interpretation, namely " $f$ is strategyproof" means that "every solution $g$ projecting onto $f$ is strategyproof".

It is convenient to discuss assignment solutions and welfarist solutions first, because $R$ is the only preference profile involved in the assignment problem $(M, W, R)$. The matching problem is more complicated because the two preference profiles $R M$ and $R W$ are in play.

Definition 3 (assignment). The assignment solution $g$ is called strategyproof $(S P)$ if for all $m \in M$ and any two preference matrices $R, R^{\prime}$ with $g(R)=Z$ and $g\left(R^{\prime}\right)=Z^{\prime}$ :

$$
\left\{R_{m^{\prime}}=R_{m^{\prime}}^{\prime} \text { for all } m^{\prime} \neq m\right\} \Rightarrow u_{m}\left(Z_{m}\right) \geq u_{m}\left(Z_{m}^{\prime}\right)
$$

The solution is called weakly groupstrategyproof (WGSP) if for all $S \subseteq M$, and any two matrices $R, R^{\prime}$ with $g(R)=Z$ and $g\left(R^{\prime}\right)=Z^{\prime}$ :

$$
\left\{R_{m^{\prime}}=R_{m^{\prime}}^{\prime} \text { for all } m^{\prime} \notin S\right\} \Rightarrow\left\{u_{m}\left(Z_{m}\right) \geq u_{m}\left(Z_{m}^{\prime}\right) \text { for some } m \in S\right\}
$$

The solution is called groupstrategyproof $(G S P)$ if under the same premises:

$$
\begin{aligned}
\left\{R_{m^{\prime}}\right. & \left.\left.=R_{m^{\prime}}^{\prime} \text { for all } m^{\prime} \notin S\right\} \text { and } u_{m}\left(Z_{m}^{\prime}\right) \geq u_{m}\left(Z_{m}\right) \text { for all } m \in S\right\} \\
& \Rightarrow\left\{u_{m}\left(Z_{m}^{\prime}\right)=u_{m}\left(Z_{m}\right) \text { for all } m \in S\right\}
\end{aligned}
$$

Clearly a priority solution is strategyproof, even weakly groupstrategyproof: for any priority ordering $\succ$ and any coalition $S$, the agent $m$ with the highest priority in $S$ is preceded in $\succ$ by a coalition $T$ disjoint from $S$, hence this agent cannot improve upon his own utility $e(T \cup\{m\})-e(T)$.

But a priority solution is not groupstrategyproof as soon as $M$ contains three agents or more: the example at the end of Section 1 can be read as an assignment problem where the highest priority agent $m_{1}$ can help the lowest priority agent $m_{3}$ at no cost to himself. It turns out that all deterministic solutions are similarly vulnerable.

Lemma 7 With three or more agents and two or more objects, no deterministic assignment solution is groupstrategyproof.

We observe now that SP is preserved by fixed probability combinations, but WGSP is not. This explains the first two statements in our next result. 
Lemma 8 The random priority assignment solution is strategyproof; it is not weakly groupstrategyproof. The same is true for the uniform assignment solution.

Theorem 2 The egalitarian assignment solution is groupstrategyproof.

Comparing Lemma 7 and Theorem 2, we find that randomization is necessary to achieve GSP in our model. We are not aware of another mechanism design problem where randomization is necessary to combine efficiency and incentive compatibility.

We turn to matching problems. There the revelation mechanism works as follows: men and women report their preferences $R M$ and $R W$ respectively, and the solution $g$ applied to the ir-reduced problem $R M \bullet R W$ implements the random allocation matrix $Z$. Here the notation $A \bullet B$ stands for the entry-byentry product of the matrices $A, B$ of the same size.

Definition 4 (matching). The matching solution g is called male-strategyproof if for all $m \in M$, and any three matrices $R M, R M^{\prime}$ and $R W$ with $g(R M \bullet R W)=$ $Z$ and $g\left(R M^{\prime} \bullet R W\right)=Z^{\prime}$ :

$$
\left\{R M_{m^{\prime}}=R M_{m^{\prime}}^{\prime} \text { for all } m^{\prime} \neq m\right\} \Rightarrow u_{m}\left(Z_{m}\right) \geq u_{m}\left(Z_{m}^{\prime}\right)
$$

The solution is called strategyproof if it is both male- and female-strategyproof.

The solution is called male-groupstrategyproof if for all $S \subseteq M$, and any three matrices $R M, R M^{\prime}$ and $R W$, with $g(R M \bullet R W)=Z$ and $g\left(R M^{\prime} \bullet R W\right)=Z^{\prime}$ :

$$
\begin{aligned}
\left\{R M_{m^{\prime}}\right. & \left.=R M_{m^{\prime}}^{\prime} \text { for all } m^{\prime} \notin S \text { and } u_{m}\left(Z_{m}\right) \geq u_{m}\left(Z_{m}^{\prime}\right) \text { for all } m \in S\right\} \\
& \Rightarrow\left\{u_{m}\left(Z_{m}\right)=u_{m}\left(Z_{m}^{\prime}\right) \text { for all } m \in S\right\}
\end{aligned}
$$

An efficient matching solution guarantees the utility 1 to every non disposable person who therefore has no incentive to misreport. From the analysis of efficiency in Lemma 6, it follows at once that if an efficient assignment solution $g^{A}$ is strategyproof, its canonical extension $g^{M}$ to matching is strategyproof as well. Similarly, if $g^{A}$ is efficient and groupstrategyproof (Definition 3), then $g^{M}$ is both male- and female-groupstrategyproof. Hence

Corollary to Lemma 8 and Theorem 2 The random priority and uniform matching solutions are strategyproof. The egalitarian matching solution is both male-groupstrategyproof and female-groupstrategyproof.

We turn to joint misreports by coalitions mixing some men and some women. A simple example shows that no efficient solution is groupstrategyproof.

Assume $M=\{m\}, W=\left\{w_{1}, w_{2}\right\}$, and both women are compatible with the unique man. Here $m$ can enforce matching $(m, w)$ by reporting that he only likes $w$, so if a matching mechanism chooses $\left(m, w_{i}\right)$ with positive probability, the coalition $m, w_{j}, j \neq i$, can manipulate to the benefit of woman $w_{j}$. 
When we only require weak groupstrategyproofness, a comparable impossibility result obtains. Its proof is more involved, however. We start with the definition of WGSP.

Definition 5 (matching). The matching solution $g$ is called weakly groupstrategyproof if for all $S \subseteq M$, all $B \subseteq W$, and any four matrices $R M, R W, R M^{\prime}, R W^{\prime}$, with $Z=g(R M \bullet R W)$ and $Z^{\prime}=g\left(R M^{\prime} \bullet R W^{\prime}\right) \bullet R M \bullet R W:$

$$
\begin{aligned}
\left\{R M_{m^{\prime}}\right. & \left.=R M_{m^{\prime}}^{\prime} \text { for all } m^{\prime} \notin S \text { and } R W_{w^{\prime}}=R W_{w^{\prime}}^{\prime} \text { for all } w^{\prime} \notin B\right\} \\
& \Rightarrow\left\{u_{m}\left(Z_{m}\right) \geq u_{m}\left(Z_{m^{\prime}}\right) \text { for some } m \in B \text { or } v_{w}\left(Z^{w}\right) \geq v_{m}\left(Z^{\prime w}\right) \text { for some } w \in B\right\}
\end{aligned}
$$

Definition 5 uses the full force of the individual rationality assumption. Following the misreport $R M^{\prime}, R W^{\prime}$ by coalitions $S$ and $B$, the solution $g$ implements the allocation matrix $\widetilde{Z}=g\left(R M^{\prime} \bullet R W^{\prime}\right)$. The pair $(m, w)$ is incompatible if $R M_{m w} \cdot R W_{m w}=0$; if $\widetilde{z}_{m w}>0$ for such a pair, the match $(m, w)$ will be implemented by $g$ with positive probability, given the reported preferences, yet it will not happen ex post because it is not voluntary for at least one of the two persons. Thus the allocation matrix actually implemented under individual rationality is $\widetilde{Z} \bullet R M \bullet R W$, as shown in Definition 5 .

Consider the following example with four men and four women. Let $m_{1}$ and $w_{1}$ like persons of the opposite gender numbered 2, 3 and $4, m_{2}$ and $w_{2}$ like persons 1,3 and 4 , while $m_{3}, m_{4}$ and $w_{3}, w_{4}$ only like person 1 . We have:



$$
\begin{aligned}
& \text { so } R=\begin{array}{lllll} 
& w_{1} & w_{2} & w_{3} & w_{4} \\
m_{1} & 0 & 1 & 1 & 1 \\
m_{2} & 1 & 0 & 0 & 0 \\
m_{3} & 1 & 0 & 0 & 0 \\
m_{4} & 1 & 0 & 0 & 0
\end{array}
\end{aligned}
$$

Consider the manipulation by $S=\left\{m_{3}, m_{4}, w_{3}, w_{4}\right\}$, where all pretend to also like person 2. We get:

$$
R M^{\prime}=\begin{array}{llll}
0 & 1 & 1 & 1 \\
1 & 0 & 1 & 1 \\
1 & 1 & 0 & 0 \\
1 & 1 & 0 & 0
\end{array}, R W^{\prime}=\begin{array}{llll}
0 & 1 & 1 & 1 \\
1 & 0 & 1 & 1 \\
1 & 1 & 0 & 0 \\
1 & 1 & 0 & 0
\end{array}, \text { so } R^{\prime}=\begin{array}{llll}
0 & 1 & 1 & 1 \\
1 & 0 & 1 & 1 \\
1 & 1 & 0 & 0 \\
1 & 1 & 0 & 0
\end{array}
$$

Note that the preference matrix $R^{\prime}$ allows a perfect match. 
Consider an efficient solution $g$ that also treats equals equally, namely for all problem $(M, W, R)$ with $g(M, W, R)=Z$ and all $m, m^{\prime}, w, w^{\prime}$ :

$$
R_{m}=R_{m^{\prime}} \Rightarrow Z_{m}=Z_{m^{\prime}} ; R^{w}=R^{w^{\prime}} \Rightarrow Z^{w}=Z^{w^{\prime}}
$$

The above property plus efficiency determine $g$ at $R$ and $R^{\prime}$ :

$$
g(R)=\begin{array}{cccc}
0 & \frac{1}{3} & \frac{1}{3} & \frac{1}{3} \\
\frac{1}{3} & 0 & 0 & 0 \\
\frac{1}{3} & 0 & 0 & 0 \\
\frac{1}{3} & 0 & 0 & 0
\end{array}, g\left(R^{\prime}\right)=\begin{array}{cccc}
0 & 0 & \frac{1}{2} & \frac{1}{2} \\
0 & 0 & \frac{1}{2} & \frac{1}{2} \\
\frac{1}{2} & \frac{1}{2} & 0 & 0 \\
\frac{1}{2} & \frac{1}{2} & 0 & 0
\end{array}
$$

Because persons 3, 4 reject, ex post, a match with person 2, the allocation matrix actually implemented is:

$$
Z^{\prime}=\begin{array}{cccc}
0 & 0 & \frac{1}{2} & \frac{1}{2} \\
0 & 0 & 0 & 0 \\
\frac{1}{2} & 0 & 0 & 0 \\
\frac{1}{2} & 0 & 0 & 0
\end{array}
$$

thus the misreport strictly benefits everyone in $S$. We conclude that $g$ violates WGSP.

A welfarist solution is $W G S P$ if all solutions projecting onto it are $W G S P$. In the Appendix, we use the same $4 \times 4$ example to prove the second statement in our next result.

Theorem 3 Assume at least 4 men and 4 women. No efficient matching solution treating equals equally is weakly groupstrategyproof. No efficient welfarist matching solution is weakly groupstrategyproof.

A priority solution that always selects a deterministic allocation matrix is an example of a WGSP efficient solution. Indeed, consider a member of the deviating coalition with the highest priority. He or she can improve only if somebody with higher priority, who was matched initially, does not get a match after manipulation. The only way this can happen is when another member of the deviating coalition pretends to like such a person, is matched to this person by some implementation of the priority solution, but refuses this match ex post. But this means that under the manipulation the utility of this last member of the deviating coalition is zero, so this person does not improve.

\section{Assignment problem: fairness and competi- tive interpretation.}

Except for the second statement in Theorem 4, valid for matching as well as assignment problems, all results in this section are for assignment problems only. 
Our first result is the competitive interpretation of the egalitarian solution for assignment problems. Consider an allocation matrix $Z, Z \in \mathcal{Z}(M, W, R)$, implementing the egalitarian profile $u^{e}$. It must assign all the objects in $R\left(T_{1}\right)$ to coalition $T_{1}$ in order to achieve the total utility $\sum_{T_{1}} u_{m}^{e}=r\left(T_{1}\right)$. All the objects in $R\left(T_{2}, W_{1}\right)=R\left(T_{2}\right) \backslash R\left(T_{1}\right)$ must be assigned to coalition $T_{2}$ to secure the utility $\sum_{T_{2}} u_{m}^{e}=r\left(T_{2}, W_{1}\right)$, given that $R\left(T_{1}\right)$ is not available. By repeating this argument we see that $Z$ must assign precisely the objects $R\left(T_{k}, W_{k-1}\right)=$ $R\left(T_{k}\right) \backslash R\left(T_{1, \ldots, k-1}\right)$ to coalition $T_{k}$ for $k=1, \ldots, K$, with the qualification that if $T_{K}=M^{a}$ (i.e., $\alpha_{K} \geq 1$ ), only $\left|M^{a}\right|$ objects in $R\left(T_{K}, W_{K-1}\right) \subseteq W^{\text {no }}$ need to be assigned to $T_{K}$.

In short, coalition $T_{k}$ receives at stage $k$ all the objects it likes among those not assigned in earlier stages.

Define now the following price vector $p$ :

$$
\begin{aligned}
& p_{w}=\frac{1}{\alpha_{k}} \text { if } w \in R\left(T_{k}, W_{k-1}\right) \quad \text { for } k=1, \ldots, K-1 \\
& p_{w}=\max \left\{1, \frac{1}{\alpha_{K}}\right\} \quad \text { if } w \in R\left(T_{K}, W_{K-1}\right)
\end{aligned}
$$

Consider the competitive demand at price $p$, for an agent $m$ in $T_{k}$ with income of 1 . This agent will buy only (a fraction of) the cheapest objects in $R_{m}$. The above discussion shows that these are precisely the objects in the non-empty set $R_{m} \cap R\left(T_{k}, W_{k-1}\right)$. They all cost $\frac{1}{\alpha_{k}}$ (or 1 if $M^{e} \neq \emptyset$ and $m \in T_{K}=M^{e}$ ) therefore with a budget of one, agent $m$ buys a fraction (probability or timeshare) $\alpha_{k}$ of his good objects.

We just proved that every egalitarian allocation matrix $Z$ ( i.e., any $Z$ implementing $\left.u^{e}\right)$ is "equal income competitive" for the price vector (11). The converse statement holds true as well.

\section{Corollary to Theorem 1}

Given are a positive price vector $p, p_{w}>0$ for all $w \in W$, and a matrix $Z, Z \in \mathcal{Z}(M, W, R)$. Suppose $Z$ is "equal income competitive" at price $p$ and income of one, namely meets the following properties:

- competitive demand for all $m$ : set $\pi_{m}=\min _{R_{m}} p_{w^{\prime}}$ then for all $w, z_{m w}>$ $0 \Rightarrow p_{w}=\pi_{m}$ and $\sum_{W} z_{m w}=\frac{1}{\pi_{m}}$

- market clearing: $Z$ is efficient, i.e., $\sum_{M \times W} z_{m w}=e(M, W, R)$

Then $Z$ is an egalitarian matrix and the price $p$ is given by (10), except perhaps for the objects in $W^{\text {no }}$ :

$$
p_{w} \geq 1 \text { for all } w \in W^{n o},
$$

with equality for at least some subset of size $\left|M^{a}\right|$ assignable to $M^{a}$

Note that the aggregate supply of problem $(M, W, R)$ is unambiguously defined if $\left|W^{n o}\right|=\left|M^{a}\right|$, in which case all objects are assigned in any efficient assignment. If $\left|W^{n o}\right|>\left|M^{a}\right|$, an efficient supply contains $W^{o}$, and a subset of $\left|M^{a}\right|$ objects in $W^{n o}$ (to be assigned to $M^{a}$ ) : there may be several such subsets. This explains the somewhat unusual form of the market clearing condition, and 
the (inconsequential) possibility of $p_{w}>1$ for some redundant objects in $W^{n o}$ : these objects are not assigned by $Z$.

We turn now to the discussion of several fairness properties, shared by our three solutions, among others. Our three solutions are fair in the basic sense of treating symmetrically all agents (equal treatment of equals: $R_{m}=R_{m^{\prime}} \Rightarrow$ $u_{m}=u_{m^{\prime}}$ ) as well as all objects (the familiar neutrality property). The microeconomic fair division literature (see e.g., Thomson and Varian [1985], Moulin [1995]) emphasizes the two specific tests of "fair share" and "no envy", as well as monotonicity properties with respect to changes in the resources or the set of agents among which the resources must be shared. The next two definitions adopt these concepts to our assignment model.

Definition 6 Fix an assignment problem $(M, W, R)$, and a utility profile $u \in$ $\mathcal{U}(M, W, R)$. We say that u guarantees a Fair Share if

$$
u_{m} \geq \min \left\{1, \frac{\left|R_{m}\right|}{|M|}\right\} \text { for all } m \in M .
$$

We say that u satisfies No Envy if:

$$
u_{m}\left(Z_{m}\right)=\sum_{R_{m}} z_{m w} \geq \sum_{R_{m}} z_{m^{\prime} w}=u_{m}\left(Z_{m^{\prime}}\right)
$$

for all $m, m^{\prime} \in M$, and all $Z$ implementing $u$

The fair share property amounts to give to agent $m$ a $\frac{1}{|M|}$-th claim on every object he likes, thus rewarding an agent whose preferences are more flexible. Notice that $\left|R_{m}\right| \geq|M|$ can only occur if $m$ is efficient, $m \in M^{a}$ : any assignment of $M \backslash m$ to $W$ leaves free at least one object in $R_{m}$, implying $e(M, W, R)=$ $e(M \backslash m, W, R)+1$, and the claim by (7). Thus Fair Share requires $u_{m}=1$ only when efficiency does too.

Definition 6 bears on utility profiles, as requested in order to apply the two properties to welfarist solutions. As in Section 6, we adopt the most demanding interpretation of the non welfarist No Envy property: a welfarist solution is non envious only if all solutions projecting onto it are non envious.

Our last two fairness properties pertain respectively to the addition of one more object, or one more person, to a given problem $(M, W, R)$.

Definition 7 The (welfarist) assignment solution $f$ is resource monotonic if for any problem $(M, W, R)$ we have:

$$
f(M, W, R) \geq f(M, W \backslash w, \widetilde{R}) \text { for all } w \in W
$$

The solution is population monotonic if for any problem $(M, W, R)$ we have: 


$$
f_{m}(M, W, R) \leq f_{m}\left(M \backslash m^{\prime}, W, \widetilde{R}\right) \text { for all } m, m^{\prime} \in M, m \neq m^{\prime}
$$

Resource and population monotonicity are hard to meet in the fair division problem with divisible goods and classical microeconomic preferences, less so in the assignment problem with strict preferences ${ }^{7}$.

Theorem 4 Each one of the egalitarian, uniform, and random priority solutions meets Fair Share and No Envy.

The egalitarian and random priority solutions are resource and population monotonic.

We do not know whether the uniform solution is monotonic, either with respect to resource or population.

Finally, we note that the second statement in Theorem 4 applies to matching problems as well. In the matching interpretation of our model, the two properties of Definition 6 correspond to the familiar complementarity properties of bilateral matching: adding one man to the matching problem $(M, W, R)$ cannot be bad news for any woman (i.e., no woman's utility decreases), and cannot be good news for any man (see Roth and Sotomayor [1990]).

If an efficient assignment solution is resource and population monotonic, its canonical extension as an (efficient) matching solution meets the complementarity properties. This follows at once from the following properties of the Gallai-Edmonds decomposition (Lemma 1): removing a non-disposable man changes neither $W^{o}$ nor $M^{d}$; removing a disposable man changes neither $W^{d}$ nor $M^{o}$.

\section{Concluding comments}

1. The properties of efficiency and strategyproofness are preserved by probabilistic selections (with fixed probabilities) among matching (or assignment) solutions. The same remark applies to all fairness properties discussed in Section 7: equal treatment of equals, neutrality, fair share, no envy, as well as the two complementarity (or monotonicity) properties. Therefore the requirements of efficiency, fairness and strategyproofness allow for an infinite set of solutions in our model; it would be interesting to know the contours of this set.

2. In the assignment problem $(M, W, R)$ the row $R_{w}$ of the preference matrix $R$ partitions $W$ into "good" objects and "bad" objects. For our results to make sense, agent $m$ must strictly prefer consuming any good object to any bad one. He must also view "no object" as at least as good as a bad object. But it is not necessary that he strictly prefers "no object" to a bad one (or that he prefers a good object to no object): it is always efficient to never give $m$ an object that he does not strictly prefer to "no object", as we do in our model.

\footnotetext{
${ }^{7}$ See Ehlers and Klaus [2000], Ehlers, Klaus and Papai [2000].
} 
Moreover, our model also accommodates the case where "no object" is not an option and, naturally, $|W| \geq|N|$. In this case efficiency implies that if $m$ is unhappy (receives an object he views as bad) no other unhappy agent views $m^{\prime} s$ object as good. Thus the distribution of objects to unhappy agents is irrelevant and the two assignment problems, without the "no object" option or with that option strictly between a good or a bad object, are isomorphic.

3 . The roommate problem is the natural generalization of bilateral matching where the (gender neutral) agents must form pairs (to share a hypothetical room). Under the assumption of dichotomous preferences and the restriction to voluntary (individually rational) matching, an ir-reduced problem is described by a pair $(N, G)$ where $N$ is the set of agents, $G$ is an undirected graph on $N$, and an edge between two agents means that they are mutually compatible.

The Gallai-Edmonds decomposition generalizes to the matching problem $(N, G)$ : see Theorem 3.2.1 in Lovasz and Plummer [1986]. In particular all inclusion maximal matchings compatible with $G$ have the same cardinality, so we can still speak of the efficiency size $e$ of an arbitrary problem. Hence for random matchings, it is still true that ex post and ex ante efficiency coincide: a random matching is efficient if and only if, with probability one, it matches exactly $2 e$ agents.

It is straightforward to extend the definition of fixed priority, random priority and uniform solutions to the roommate problem. One checks just as easily that these solutions are strategyproof. Therefore, the latter two solutions are efficient, strategyproof and fair (in the sense of Equal Treatment of Equals).

As in bilateral matching, weak groupstrategyproofness is out of reach in the roommate problem, if we also insist on efficiency and fairness. Unlike in bilateral matching, this fact does not depend upon the ex post rejection of matches. Consider the three person problem where each one of agents 1, 2 and 3 like the other two, so that $G$ is the complete graph. An efficient solution treating equals equally must match each pair $\{i, j\}$ with probability $\frac{1}{3}$, resulting in the utility $\frac{2}{3}$ for each. Now if 1 and 2 both report that they only like each other, they are matched with probability 1 (by efficiency). 


\section{References}

[1] Abdulkadiroglu, A. and T. Sönmez, 1998. "Random serial dictatorship and the core from random endowments in house allocation problems," Econometrica, 66, 689-701.

[2] Bogomolnaia, A. and R. Deb, 2000. "Housing market with indifferences: the priority method," mimeo Southern Methodist University.

[3] Bogomolnaia, A. and H. Moulin, 1999. "A simple random assignment problem with a unique solution," forthcoming Economic Theory.

[4] Bogomolnaia, A. and H. Moulin, 2000. "A new solution to the random assignment problem," forthcoming Journal of Economic Theory.

[5] Dubins L. E. and D. A. Freedman 1981. "Machiavelli and the Gale-Shapley algorithm," American Mathematical Monthly, 88, 485-94.

[6] Dutta, B., 1990. "The egalitarian solution and reduced game properties in convex games," International Journal of Game Theory, 19:153-169.

[7] Dutta, B. and D. Ray, 1989. "A concept of egalitarianism under participation constraints," Econometrica, 57, 615-635.

[8] Ehlers, L. and B. Klaus, 2000. "Coalitional strategyproof and resource monotonic solutions for multiple assignments problems," forthcoming, Social Choice and Welfare.

[9] Ehlers, L., B. Klaus and S. Papai, 2000. "Strategyproofness and population monotonicity for house allocation problems," mimeo, University of Nebraska at Lincoln.

[10] Gale, D and M. Sotomayor, 1985. "Ms. Machiavelli and the stable matching problem," American Mathematical Monthly, 92, 261-8.

[11] Hylland, A. and R. Zeckhauser, 1979. "The efficient allocation of individuals to positions," Journal of Political Economy, 91, 293-313.

[12] Lovasz, L. and M. D. Plummer, 1986. Matching Theory, Annals of Discrete Mathematics, \#29, Amsterdam: North Holland.

[13] Moulin, H., 1988. Axioms of Cooperative Decision Making, Monograph of the Econometric Society, Cambridge: Cambridge University Press.

[14] Moulin, H., 1995. Cooperative Microeconomics: A Game Theoretic Introduction, Princeton, New Jersey: Princeton University Press.

[15] Papai, S., 2000. "Strategyproof assignment by hierarchical exchange," Econometrica, 68, 6, 1403-1434.

[16] Roth, A., 1982. "The economics of matching: stability and incentives," Mathematics of Operations Research, 7, 617-28. 
[17] Roth, A. and M. Sotomayor, 1990. Two-sided matching, Cambridge: Cambridge University Press.

[18] Shapley, L., 1971. "Core of convex games," International Journal of Game Theory, 1, 11-26.

[19] Sprumont, Y, 1990. "Population monotonic allocation schemes for cooperative games with transferable utility," Games and economic Behavior, 2, 378-394.

[20] Svensson, L. G., 1994. "Queue allocation of indivisible goods," Social Choice and Welfare, 11, 323-330.

[21] Svensson, L. G., 1999. "Strategy-proof allocation of indivisible goods," Social Choice and Welfare, 16, 557-567.

[22] Thomson, W. and H. Varian, 1985. "Theories of justice based on symmetry," in L. Hurwicz, D. Schmeidler and H. Sonnenschein eds., Social Goals and Social Organization, Cambridge University Press.

[23] Wako, J., 1991. "Some properties of weak domination in an exchange market with indivisible goods," The Economics Studies Quarterly, 42, 303-314.

[24] Zhou, L., 1990. "On a conjecture by Gale about one-sided matching problems," Journal of Economic Theory, 52, 123-135. 


\section{Appendix: Proofs}

\section{Proofs for Section 4}

Lemmas 1-3 can all be retrieved from matching theory upon translating our economic terminology into the graph-theoretical terminology of matching. See in particular in Lovasz and Plummer [1986], Chapter 3. For the sake of completeness, we give a sketch of the proof.

\section{Lemma 1:}

Interpret our problem as an assignment problem for men. For any subset $B$ of objects, $B \subseteq W$, consider the property $Q(B)$ " $R^{B}$ can be assigned to $B$ ". The property is clearly stable by union: let $W^{\text {no }}$ be the largest set possibly empty - for which $Q(B)$ holds. Set $M^{a}=R^{W^{n o}}$. We check that $W^{o}=W \backslash W^{n o}$ is overdemanded by $M^{d}=M \backslash M^{a}$, in the sense of property (4). If $W^{o}=\emptyset \Leftrightarrow M^{d}=\emptyset$ we are done, so we assume $W^{o} \neq \emptyset$.

For any $w \in W^{o}, R^{w} \cap M^{d}=\emptyset$ or $R^{w} \cap M^{d}=\{m\}$ implies $Q\left(W^{n o} \cup\{w\}\right)$, contradicting the definition of $W^{n o}$. Therefore (4) holds for any singleton $\{w\}$. Suppose $B$, a subset of $W^{o}$, fails (4) but every proper subset of $B$ meets (4). As $B$ contains at least two objects, we can pick $B^{\prime} \subseteq B$, non empty and of size $|B|-1$. Because $B^{\prime}$ meets (4) we have:

$$
|B|=\left|B^{\prime}\right|+1 \leq\left|R^{B^{\prime}} \cap M^{d}\right| \leq\left|R^{B} \cap M^{d}\right| \Rightarrow\left|R^{B} \cap M^{d}\right|=|B|
$$

By Hall's theorem, $B$ can be assigned to $R^{B} \cap M^{d}$.

Because these two sets are of the same size, $R^{B} \cap M^{d}$ can be assigned to $B$. Now $R^{W^{n o} \cup B}=M^{a} \cup\left(R^{B} \cap M^{d}\right)$ can be assigned to $W^{n o} \cup B$ and we have reached a contradiction.

To prove that the decomposition is unique, check that properties $i)$, ii), iii) imply that $W^{n o}$ is the largest subset $B$ meeting $Q(B)$.

To prove the lemma for matching problems, consider $\left(M^{a}, W^{n o}, \widetilde{R}\right)$ as an assignment problem for women. We obtain the unique decomposition of $M^{a}$ into $M^{o}$ and $M^{p}$, and of $W^{n o}$ into $W^{d}$ and $W^{p}$.

\section{Lemma 2:}

As before, it is enough to check the lemma for assignment problem, and to apply it then in turns to male and female assignments. By Lemma $1, e$ is the largest number of agents that can be assigned in any assignment $\mu$. Hence the "if" statement: $\mu$ is efficient if it assigns $e$ agents.

To prove "only if", we show by induction on $|M|$ the following property. Pick a coalition $S$ that is assigned by some $\mu \in \mathcal{A}(M, W, R)$ and such that $|S|<e$. Then there exists a larger $S^{\prime}$ containing $S$, that can also be assigned. The property is obvious for $|M|=1,2$.

Notice that $e \leq|M|$ therefore $|S|<|M|$, and let 1 be an agent outside $S$. If $|S|<e^{\prime}=e(M \backslash 1, W, \widetilde{R})$, the induction hypothesis gives the desired conclusion. As $e^{\prime}$ cannot be less than $e-1$, we are left with the following situation: 


$$
e(M \backslash 1, W, \widetilde{R})=|S|<e(M, W, R)=e(M \backslash 1, W, \widetilde{R})+1
$$

Since $e$ does not increase when we add a disposable agent, it must be that $1 \in M^{a}$. Now $\mu$ assigning $S$ (see above) must assign $S \cap M^{d}$ to $W^{o}$ (no one in $M^{d}$ likes any object in $\left.W^{n o}\right)$, and on the other hand $\left(S \cap M^{a}\right) \cup\{1\}$ can be assigned to $W^{n o}$ : therefore $S \cup\{1\}$ can be assigned.

\section{Lemma 3:}

Follows immediately from Lemma 2, given that the exclusion of a nondisposable man changes neither the set of overdemanded women nor that of disposable men.

Lemma 4:

a) Submodularity of $S \rightarrow e(S, W, \widetilde{R})$. It is enough to show the inequality:

$$
e\left(T+m+m^{\prime}\right)+e(T) \leq e(T+m)+e\left(T+m^{\prime}\right)
$$

for all $T \subseteq M$, and $m, m^{\prime}$ distinct outside $T$. We use the lighter notation $T+m+$ $m^{\prime}$ instead of $T \cup\left\{m, m^{\prime}\right\}$, and so on. Because $e(S+k)-e(S)=0$ or 1 for all $S$ and $k$, inequality (14) is clear if $e(T+m)=e(T)+1$ or $e\left(T+m^{\prime}\right)=e(T)+1$. All we need to prove is that the configuration:

$$
e(T+m)=e\left(T+m^{\prime}\right)=e(T) ; e\left(T+m+m^{\prime}\right)=e(T)+1
$$

is impossible. We assume (15) and derive a contradiction. By (6), $m$ and $m^{\prime}$ are both efficient in $\left(T+m+m^{\prime}, W, \widetilde{R}\right)$.

The following claim about the decomposition of Lemma 1 is easy to prove: starting from problem $(M, W, R)$ with the efficient set of men $M^{a}=M^{o} \cup M^{p}$, if the set $M^{\prime}$ obtains from $M$ by taking away some agents in $M^{a}$, the efficient set of men for $\left(M^{\prime}, W, \widetilde{R}\right)$ is simply $M^{\prime} \cap M^{a}$, and $e\left(M^{\prime}, W, \widetilde{R}\right)=e(M, W, R)-$ $\left|M \backslash M^{\prime}\right|$.

Applying the claim to $M=T+m+m^{\prime}$ and $M^{\prime}=T$, we get $e(T)=$ $e\left(T+m+m^{\prime}\right)-2$, contradicting (15).

b) Submodularity of $B \rightarrow e(M, B, \widetilde{R})$ : same as above, upon exchanging the roles of $M$ and $W$.

c) Supermodularity of $(S, B) \rightarrow e(S, B, \widetilde{R})$

It is enough to show:

$$
e(S+m, B+w)+e(S, B) \geq e(S, B+w)+e(S+m, B)
$$

whenever $m$ is outside $S$ and $w$ outside $B$. This is obvious if $e(S, B)=e(S+$ $m, B)$, therefore we only need to prove that for all $S, B, m \notin S$ and $w \notin B$ : 


$$
e(S+m, B)=e(S, B)+1 \Rightarrow e(S+m, B+w)=e(S, B+w)+1
$$

Under the premises of (16), men $m$ is efficient in $(S+m, B, \widetilde{R})$ (by (6)) and the desired conclusion follows yet another simple fact about the decomposition of Lemma 1 (the proof of which is left to the reader): starting with problem $(M, W, R)$ with the efficient set of men $M^{a}$, if we add one new woman to $W$, the efficient set of men can only grow; formally, the mapping $W \rightarrow M^{a}$ is inclusion monotonic.

\section{Lemma 5:}

The easy proof of the first statement is omitted. The second statement is proved by Bogomolnaia and Deb [2000] in the more general context with complete and transitive preferences.

\section{Lemma 6:}

a) Statement $i$ : Clear from Lemma 2 and the discussion before Lemma 6 .

b) Statement $i i$ :

Let $\mathcal{Z}_{0}$ be the subset of $\mathcal{Z}(M, W, R)$ containing the allocation matrices of deterministic assignments, and $\mathcal{Z}_{0}^{e}$ be the subset of $\mathcal{Z}_{0}$ corresponding to efficient deterministic assignments. By Lemma $2, \max _{\mathcal{Z}_{0}} \sum_{M \times W} z_{m w}=e$, and $\mathcal{Z}_{0}^{e}$ is precisely the set of solutions of this maximization program. Now $\mathcal{Z}$ is the convex hull of $\mathcal{Z}_{0}$, and by statement $i$, its subset $\mathcal{Z}^{e}$ of efficient allocation matrices is the convex hull of $\mathcal{Z}_{0}^{e}$. Therefore $\mathcal{Z}^{e}$ is the solution set for the program $\max _{\mathcal{Z}} \sum z_{m w}$.

c) Statement iii: Follows immediately from Lemmas 1,2 and the discussion after them.

d) Statement iv:

Check it first for assignment problem. For any coalition $S$ and any deterministic assignment $\mu, \mu \in \mathcal{A}(M, W, R)$, with associated utility profile $u$, the sum $\sum_{S} u_{m}$ is the number of agents in $S$ assigned by $\mu$. Therefore if $\mu$ is efficient its utility profile meets system (8). By linearity, so does the utility profile of any efficient random assignment.

To prove the converse statement, let $u \in[0,1]^{M}$ be a solution of system (8). By a classical result about submodular (concave) cooperative games (Shapley [1971]), $u$ is a convex combination of marginal contribution profiles $u^{\succ}$. For a strict ordering $\succ$ of $M$, this vector is defined by:

$$
u_{m}^{\succ}=e(T \cup\{m\}, W, \widetilde{R})-e(T, W, \widetilde{R}) \text { for all } m
$$

where $T$ is the set of agents preceding $m$ in $\succ$.

By Lemma $5, u^{\succ}$ is the utility profile of the (efficient) $\succ$ priority assignment. Thus $u$ is the utility profile of an efficient random assignment.

Statement $i v$ for matching problems follows now from property iii.

\section{Proofs for Section 5: Theorem 1}


Step 1 Preliminary result.

$\overline{\text { Let } f}$ be a non negative submodular function defined over the non-empty subsets of $M$. Fix a coalition $S, S \subseteq M$, and consider the program of minimizing $\frac{f(T)}{|T|}$ over all non-empty subsets of $S$. It is easy to check that the set of its solutions is stable by union, therefore the largest solution is well-defined; this set is denoted $\arg \min _{S} \frac{f(T)}{T T}$.

For any subset $B$ of $W$, the function $T \rightarrow r(T, B)$ is obviously submodular, therefore the sequence $T_{k}, k=1,2 \ldots$ is well-defined by (9).

\section{Step 2. Another algorithm}

We apply the above result to the submodular function $T \rightarrow e(T, W, \widetilde{R})$ denoted simply $e(T)$. We construct inductively two sequences $\beta_{k}, S_{k}, k=1,2 \ldots$ by an algorithm similar to (9), introduced by Dutta and Ray [1989]:

$$
\begin{aligned}
& N_{0}=M, \quad e_{0}=e ; \text { next for } k=1,2 \ldots \\
& \beta_{k}=\min _{N_{k-1}} \frac{e_{k-1}(T)}{|T|} ; \quad S_{k}=\arg \min _{N_{k-1}} \frac{e_{k-1}(T)}{|T|} \\
& N_{k}=M \backslash S_{1, \ldots, k} ; \quad e_{k}(T)=e\left(T \cup S_{1, \ldots, k}\right)-e\left(S_{1, \ldots, k}\right)
\end{aligned}
$$

By construction, the sets $S_{k}$ are disjoint and there is a step $L$ such that $S_{1}, \ldots S_{L}$ partition $M$, at which point the sequence stops.

Dutta and Ray's egalitarian solution for the submodular (concave) cooperative game $T \rightarrow e(T)$ is the utility profile $x, x_{m}=\beta_{k}$ if $m \in S_{k}, k=1, \ldots, L$. They show that $x$ is in the core of the game $(M, e)$, and Lorenz dominates every other utility profile in the core. Translated with our terminology, the core property is system (7), thus the result says that $x$ is an efficient profile, $x \in \mathcal{U}^{e}(M, W, R)$, and it Lorenz dominates every other feasible utility profile (even inefficient ones).

For the sake of completeness, we prove below that $x$ is in $\mathcal{U}^{e}(M, W, R)$, and that is maximizes the leximin ordering over this set. We also show that the sequence $\beta_{k}$ is strictly increasing. Then we proceed to check that $\left(\beta_{k}, S_{k}\right)$ and $\left(\alpha_{k}, T_{k}\right)$ are two sequences of the same length, $K=L$, and that they coincide with the possible exception of the last term.

The equality $\sum_{M} x_{m}=e(M)$ is clear from (17). Feasibility of $x$ requires $\sum_{S} x_{m} \leq e(S)$ for all $S$. Suppose $S \subseteq S_{1}$, then $\sum_{S} x_{m}=|S| \cdot \beta_{1} \leq e(S)$ by definition of $\beta_{1}$. Suppose $S \subseteq S_{1,2}$, and set $S^{i}=S \cap S_{i}, i=1,2$. By definition of $\beta_{2}$ and submodularity of $e$ :

$$
\beta_{2} \leq \frac{e\left(S^{2} \cup S_{1}\right)-e\left(S_{1}\right)}{\left|S^{2}\right|} \leq \frac{e(S)-e\left(S^{1}\right)}{\left|S^{2}\right|}
$$

Combining this with $\beta_{1} \leq \frac{e\left(S^{1}\right)}{\left|S^{1}\right|}$ gives $\beta_{1} \cdot\left|S^{1}\right|+\beta_{2} \cdot\left|S^{2}\right| \leq e(S)$, as desired. And so on inductively. 
Next we check that $\beta_{k}$ is strictly increasing. Note first that $e(T) \leq|T|$ for all $T$, implying $\beta_{k} \leq 1$ for all $k$. The inequality $\beta_{k}<\beta_{k+1}$ means $\beta_{k}<\frac{e_{k}(T)}{|T|}$ for all $T \subseteq N_{k}$. Assume the latter fails for some $T$, namely:

$$
\beta_{k}=\frac{e_{k-1}\left(S_{k}\right)}{\left|S_{k}\right|} \geq \frac{e_{k}(T)}{|T|}
$$

Both ratios above are at most 1 therefore:

$$
\beta_{k} \geq \frac{e_{k-1}\left(S_{k}\right)+e_{k}(T)}{\left|S_{k}\right|+|T|} \geq \frac{e_{k-1}\left(T \cup S_{k}\right)}{\left|T \cup S_{k}\right|}
$$

But $S_{k}$ is the largest solution of min $\frac{e_{k-1}(T)}{|T|}$, contradiction.

It is now easy to check that $x$ maximizes the leximin ordering over $\mathcal{U}^{e}$. Suppose $y$, another profile in $\mathcal{U}^{e}$, is leximin preferred to $x$. Then for all $m \in$ $S_{1}, y_{m} \geq x_{1}^{*}=x_{m}$, and moreover:

$$
e\left(S_{1}\right) \geq \sum_{S_{1}} y_{m} \geq \sum_{S_{1}} x_{m}=e\left(S_{1}\right)
$$

therefore $y$ and $x$ coincide on $S_{1}$. Next:

$$
\text { for all } m \in S_{2} \quad y_{m} \geq y_{\left|S_{1}\right|+1}^{*} \geq x_{\left|S_{1}\right|+1}^{*}=x_{m}
$$

and moreover:

$$
e\left(S_{1} \cup S_{2}\right) \geq \sum_{S_{1} \cup S_{2}} y_{m} \geq \sum_{S_{1} \cup S_{2}} x_{m}=e\left(S_{1} \cup S_{2}\right)
$$

so that $y$ and $x$ coincide on $S_{1} \cup S_{2}$. And so on.

For the sake of brevity, we refer the reader to Theorem 3 in Dutta and Ray [1989] for a proof of the fact that $x$ is Lorenz dominant in $\mathcal{U}^{e}$.

Step 3. Equality of the two sequences

We check by induction that, as long as $\beta_{k}$ remains strictly below 1 , the two algorithms (9) and (17) coincide. More precisely we prove the following property $\mathcal{P}(k)$ by induction on $k$ :

$\beta_{k}<1 \Rightarrow\left\{\right.$ for $\left.t=1, \ldots, k: \alpha_{t}=\beta_{t}, T_{t}=S_{t}\right\} ; e\left(T_{1, \ldots, k}\right)=r\left(T_{1, \ldots, k}\right), T_{1, \ldots, k} \subseteq M^{d}$

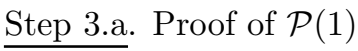


Assume we know $e\left(S_{1}\right)=r\left(S_{1}\right)$. This implies $\beta_{1}=\frac{r\left(S_{1}\right)}{\left|\left(S_{1}\right)\right|} \geq \alpha_{1}$. On the other hand $e(T) \leq r(T)$ for all $T$ implies $\beta_{1} \leq \alpha_{1}$, so that $\beta_{1}=\alpha_{1}$ and $S_{1} \subseteq T_{1}$. Next we have $\beta_{1}=\alpha_{1}=\frac{r\left(T_{1}\right)}{\left|\left(T_{1}\right)\right|} \geq \frac{e\left(T_{1}\right)}{\left|\left(T_{1}\right)\right|}$, implying $T_{1} \subseteq S_{1}$. Thus the equality $e\left(S_{1}\right)=r\left(S_{1}\right)$ is enough to conclude $\alpha_{1}=\beta_{1}, S_{1}=T_{1}$.

We assume $e\left(S_{1}\right)<r\left(S_{1}\right)$ and show a contradiction of the assumption $\beta_{1}<$ 1. In the Gallai-Edmonds decomposition of $\left(S_{1}, W, \widetilde{R}\right), S_{1}^{a}$ is non-empty (otherwise $e\left(S_{1}\right)=r\left(S_{1}\right)$, see Lemmas 1,2$)$. If $S_{1}^{d}$ is empty, $e\left(S_{1}\right)=\left|S_{1}\right| \Rightarrow \beta_{1}=1$. Thus both $S_{1}^{a}$ and $S_{1}^{d}$ are non-empty and we have, with the notations of Lemmas 1, 2:

$$
\frac{e\left(S_{1}^{d}\right)}{\left|S_{1}^{d}\right|}=\frac{\left|W^{o}\right|}{\left|S_{1}^{d}\right|}<\frac{\left|W^{o}\right|+\left|S_{1}^{a}\right|}{\left|S_{1}^{d}\right|+\left|S_{1}^{a}\right|}=\frac{e\left(S_{1}\right)}{\left|S_{1}\right|}
$$

where the inequality follows from $\left|W^{o}\right|<\left|S_{1}^{d}\right|$, namely the overdemanded character of $W^{o}$. We have reached a contradiction of the definition of $\beta_{1}$.

To conclude the proof of $\mathcal{P}(1)$, we check $T_{1}=S_{1} \subseteq M^{d}$. We have $e(T)=|T|$ for all $T \subseteq M^{a}$ therefore $T_{1} \subseteq M^{a}$ is impossible. Set $T^{*}=T_{1} \backslash M^{a}, q=$ $\left|T_{1} \cap M^{a}\right|$, so that $e\left(T_{1}\right)=e\left(T^{*}\right)+q$ (by Lemma 1 and Figure 1$)$.

If $q$ is not zero, we have:

$$
\frac{e\left(T^{*}\right)}{\left|T^{*}\right|}=\frac{e\left(T_{1}\right)-q}{\left|T_{1}\right|-q}<\frac{e\left(T_{1}\right)}{\left|T_{1}\right|}
$$

again a contradiction of the definition of $\alpha_{1}=\beta_{1}$.

Step 3.b. Induction step

$\overline{\text { Assume } \mathcal{P}}(k-1)$ and $\beta_{k}<1$. Consider an arbitrary coalition $T \subseteq M_{k-1}$. By $\mathcal{P}(k-1), e\left(T_{1, \ldots, k-1}\right)=r\left(T_{1, \ldots, k-1}\right)$, which means that all objects in $R\left(T_{1, \ldots, k-1}\right)$ can be assigned to $T_{1, \ldots, k-1}$. Clearly this implies:

$$
\begin{aligned}
e\left(T \cup T_{1, \ldots, k-1}\right) & =e\left(T_{1, \ldots, k-1}\right)+e\left(T, W \backslash R\left(T_{1, \ldots, k-1}\right)\right) \\
& \Leftrightarrow e_{k-1}(T)=e\left(T, W_{k-1}\right)
\end{aligned}
$$

We see now that the $k$-th step in our two algorithms are to minimize, respectively, $\frac{e\left(T, W_{k-1}\right)}{|T|}$ and $\frac{r\left(T, W_{k-1}\right)}{|T|}$. Upon changing $W$ to $W_{k-1}$, the same argument as in Step 3.a shows now: $\alpha_{k}=\beta_{k}, T_{k}=S_{k}$, as well as $e\left(T_{k}, W_{k-1}\right)=$ $r\left(T_{k}, W_{k-1}\right)$. Combining this with (19) and $\mathcal{P}(k-1)$, we get:

$e\left(T_{1, \ldots, k-1}\right)=e\left(T_{1, \ldots, k-1}\right)+e\left(T_{k}, W_{k-1}\right)=r\left(T_{1, \ldots, k-1}\right)+r\left(T_{k}, W_{k-1}\right)=r\left(T_{1, \ldots, k-1}\right)$

To complete Step 3.b it remains to show $T_{1, \ldots, k} \subseteq M^{d}$. $>$ From $T_{1, \ldots, k-1} \subseteq$ $M^{d}$ we get $R\left(T_{1, \ldots, k-1}\right) \subseteq W^{o}$ implying that $W_{k-1}$ contains $W^{n o}$. Therefore 
$e\left(T, W_{k-1}\right)=|T|$ for all $T \subseteq M^{a}$. We see now that $T_{k}$ is not contained in $M^{a}$ and set $T^{*}=T_{k} \backslash M^{a}, q=\left|T_{k} \cap M^{a}\right|$. Check that $e\left(T_{k}, W_{k-1}\right)=e\left(T^{*}, W_{k-1}\right)+q$ and derive a contradiction if $q$ is not zero, as in (18).

\section{Step 4. Proof of Theorem 1}

The sequence $\beta_{k}$ increases, and $\beta_{k} \leq 1$. If $T_{1, \ldots, K}=M$ and $\beta_{K}<1, \mathcal{P}(K)$ establishes the full equality of the two algorithms, and we are done. Incidentally, in this case $M=M^{d}$ (as $T_{1, \ldots, K} \subseteq M^{d}$ ), i.e., all objects must be overdemanded in problem $(M, W, R)$. If $\beta_{K-1}<1=\beta_{K}$, then (19) is valid and $\beta_{K}=1$ reads $e\left(T, W_{K-1}\right)=|T|$ for all $T \subseteq N_{K-1}$. Thus $S_{K}=M_{K-1}$ and the algorithm (17) stops there. Moreover $N_{K-1}=M_{K-1}$ can be assigned to $W_{K-1}$, and on the other hand only the agents in $M_{K-1}$ like $W_{K-1}$ :

$$
R\left(M \backslash M_{K-1}\right)=R\left(T_{1, \ldots, K-1}\right)=W \backslash W_{K-1}
$$

Recall that $W^{n o}$ is the largest subset $B$ of $W$ such that $R^{B}$ can be assigned to $B$ (see the proof of Lemma 1): therefore $M^{a}$ contains $M_{K-1}$. By $\mathcal{P}(K-1)$, $M^{d}$ contains $M \backslash M_{K-1}$, thus both inclusions are equalities: $T_{1, \ldots, K-1}=M^{d}$, $S_{K}=M^{a}$. Moreover $W_{K-1}=W^{n o}$.

Turning to the K-th step of algorithm (9) we see that $\alpha_{K} \geq 1$, because $N_{K-1}=N^{e}$ is assignable to $A_{K-1}=A^{e}$. Therefore the algorithm (9) stops here too. This completes the proof of Theorem 1, and of the properties discussed immediately after its statement.

\section{Proofs for section 6}

\section{Lemma 7:}

It is enough to consider $|M|=3,|W|=2$. Let a deterministic assignment mechanism among $M=1,2,3$ and $W=a, b$ be groupstrategyproof and efficient. We derive a contradiction by considering the following eight different preference matrices $R$ :

\begin{tabular}{|l|l|l|}
\hline & $a$ & $b$ \\
\hline$R_{1}$ & 1 & 1 \\
\hline$R_{2}$ & 1 & 1 \\
\hline$R_{3}$ & 1 & 1 \\
\hline
\end{tabular}

$[1]$

\begin{tabular}{|l|l|}
\hline 1 & 0 \\
\hline 1 & 1 \\
\hline 1 & 1 \\
\hline
\end{tabular}

[2];

\begin{tabular}{|l|l|}
\hline 1 & 0 \\
\hline 1 & 0 \\
\hline 0 & 1 \\
\hline
\end{tabular}

[3];

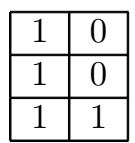

\begin{tabular}{|l|l|}
\hline 1 & 1 \\
\hline 1 & 0 \\
\hline 1 & 1 \\
\hline
\end{tabular}

$[5]$

\begin{tabular}{|l|l|}
\hline 0 & 1 \\
\hline 1 & 0 \\
\hline 1 & 1 \\
\hline
\end{tabular}

$[6]$
$[7]$

\begin{tabular}{|l|l|}
\hline 1 & 1 \\
\hline 1 & 0 \\
\hline 0 & 1 \\
\hline
\end{tabular}

The efficiency size of all eight problems is 2 . We write an assignment $(i, j)$ to indicate that object $a$ goes to agent $i$ and $b$ to $j$. We write a two-person coalition simply as $i j$.

Suppose, without loss of generality, that our mechanism chooses $(1,2)$ at [1]. Then it must choose $(1,2)$ at [2] : if $u_{1}=0$ or $u_{2}=0$ at [2], coalition 
12 can manipulate at [2] by $R^{\prime}=[1]$. Consider [3] next: the only two efficient assignments are $(1,3)$ and $(2,3)$; if $(2,3)$ is selected, 23 manipulates at [2] by $R^{\prime}=[3]$, hence $(1,3)$ is chosen at [3]. Then $(1,3)$ is chosen at [4] as well: the other efficient choice $(2,3)$ allows 13 to manipulate at [4] by reporting [3] (and 23 to manipulate at [3] by [4]!). Now at [5] the efficient coalition 13 must be chosen (by way of $(1,3)$ or $(3,1)$ ) or 13 could manipulate at [5] by $R^{\prime}=[4]$.

Consider [6]: if $(2,1)$ is selected, 12 manipulates at [5] by [6]; if $(2,3)$ is selected, 13 manipulates at [6] by $R^{\prime}=[4]$. Thus $(3,1)$ is selected. Now if $(2,3)$ is selected at [7], 23 manipulates at [6] by [7]; therefore, by efficiency, $(2,1)$ is chosen at [7]. Consider [8]: if $(1,3)$ or $(2,3)$ is selected, 12 manipulates at [8] by [7], thus $(2,1)$ is chosen at [8] as well. Recall that $(1,3)$ is chosen at [3]: therefore 13 manipulates at [8] by [3], and we have reached a contradiction.

Lemma 8:

Step 1: both solutions are strategyproof.

Consider the uniform solution. We fix $(M, W, R), m \in M$, and a misreport $R_{m}^{\prime}$. The matrix $R^{\prime}$ obtains from $R$ by replacing $R_{m}$ by $R_{m}^{\prime}$. We write $E_{m}^{\prime}=$ $E_{m}\left(M, W, R^{\prime}\right)$. Note that $E_{-m}^{\prime}=E_{-m}$. Pick a coalition $S \in E_{m}^{\prime}$, such that any $\mu$ assigning $S$ has $\mu(m) \notin R_{m}$. Then $m$ gets zero utility when an assignment corresponding to this coalition is selected. On the other hand, if $S \in E_{m}^{\prime}$ is such that at least one $\mu$ assigning $S$ has $\mu(m) \in R_{m}$, then $S \in E_{m}$. Therefore for any matrix $Z^{\prime}$ implementing the uniform solution at $R^{\prime}$ we have:

$$
u_{m}\left(Z_{m}^{\prime}\right) \leq \frac{\left|E_{m}^{\prime} \cap E_{m}\right|}{\left|E^{\prime}\right|}=\frac{\left|E_{m}^{\prime} \cap E_{m}\right|}{\left|E_{-m}\right|+\left|E_{m}^{\prime}\right|} \leq \frac{\left|E_{m}\right|}{\left|E_{-m}\right|+\left|E_{m}\right|}=u_{m}\left(Z_{m}\right)
$$

Random priority: a fixed priority mechanism is clearly strategyproof, and this property is preserved by convex combinations.

Step 2: both solutions fail weak groupstrategyproofness.

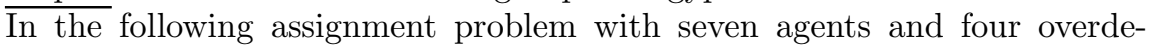
manded objects we compute the random priority solution:

$R_{1}=\{a, b, d\} ; R_{2}=\{b, c, d\} ; R_{3}=\{a, c, d\} ; R_{4}=\{a\} ; R_{5}=\{b\} ; R_{6}=\{c\} ; R_{7}=\{d\}$

The symmetries of the problem imply that agents $1,2,3$ have the same utility $x$. We compute the expected value of $u_{1}+u_{2}+u_{3}=Y$ when the priority ordering $\succ$ is drawn at random with uniform probability.

If the first four in $\succ$ include $1,2,3$ then $Y=3$. If the first four in $\succ$ include two among 1,2,3 then $Y=2$ because in this case the first four always form an efficient coalition. If the first four in $\succ$ include one among $1,2,3$, say 1 , then $Y=1$ if the other three are 456,467 or 567(because the first four are then efficient). If the other three are 457 , objects $a, b, d$ are taken by three among the first four, agent 1 among these three with probability $\frac{3}{4}$; and object $c$ is taken by the first among 2,3 , and 6 . Therefore the expected value of $Y$ when the first four include one of $1,2,3$ is $\frac{3}{4}+\frac{1}{4}\left(\frac{3}{4}+\frac{2}{3}\right)=\frac{53}{48}$. Finally $Y=0$ if the first four in $\succ$ are $4,5,6,7$. Thus we have: 


$$
3 x=\frac{1}{35}\left(4 \cdot 3+18 \cdot 2+12 \cdot \frac{53}{48}+0\right)=1.75 \quad \Rightarrow x=0.583
$$

Next suppose that the agents 1,2,3 jointly misreport by $R_{1}^{\prime}=\{a, d\}, R_{2}^{\prime}=$ $\{b, d\}, R_{3}^{\prime}=\{c, d\}$. We compute the expected value of $Y^{\prime}=u_{1}+u_{2}+u_{3}$ according to the number $q$ of agents in 1,2,3 among the first four in $\succ$ :

$$
q=3 \Rightarrow E Y^{\prime}=3 ; q=2 \Rightarrow E Y^{\prime}=2 \frac{1}{18} ; q=1 \Rightarrow E Y^{\prime}=\frac{13}{12} ; q=0 \Rightarrow Y^{\prime}=0
$$

For instance, suppose 1,2 are among the first four $(q=2)$. Then if the other two contain 6 (probability $\frac{1}{2}$ ), the first four form an efficient coalition and $Y^{\prime}=2$; if the other two are 45 , then $E\left(u_{1}+u_{2}\right)=\frac{3}{2}$ (every subset of three from 1245 can be assigned to $a b d$ ) and that of $u_{3}$ is $\frac{1}{2}$; if the other two are 47 or 57 , then $E\left(u_{1}+u_{2}\right)=1+\frac{2}{3}$ (either agent 1 gets $a$ for sure, or 2 gets $b$ for sure) and $E\left(u_{3}\right)=\frac{1}{2}$. We omit the similar computation of $E Y^{\prime}$ when $q=1$. Summing up to compute the expected utility $x^{\prime}$ of $i=1,2,3$ after the misreport:

$$
3 x^{\prime}=\frac{1}{35}\left(4 \cdot 3+18 \cdot 2 \frac{1}{18}+12 \cdot \frac{13}{12}\right)=1.77 \Rightarrow x^{\prime}=0.590
$$

The same manipulation in the same problem is also profitable under the uniform mechanism. In the truthful profile, out of 35 coalitions of four agents, only 1457,2567 and 3467 are not efficient therefore $u_{i}=\frac{19}{32}$ for $i=1,2,3$. After the misreport, there are only 20 efficient coalitions:

\begin{tabular}{|l|l|l|l|l|}
\hline$a$ & $b$ & $c$ & $d$ & $\#$ coalitions \\
\hline 1 or 4 & 2 or 5 & 3 or 6 & 7 & 8 \\
\hline 4 & 2 or 5 & 3 or 6 & 1 & 4 \\
\hline 1 or 4 & 5 & 3 or 6 & 2 & 4 \\
\hline 1 or 4 & 2 or 5 & 6 & 3 & 4 \\
\hline
\end{tabular}

Therefore $u_{i}^{\prime}=\frac{3}{5}>\frac{19}{32}$ for $i=1,2,3$.

\section{Theorem 2:}

We fix two profiles $R, R^{\prime}$, respectively the true and the false profile, and two allocation matrices $Z \in \varphi(M, W, R), Z^{\prime} \in \varphi\left(M, W, R^{\prime}\right)$. We define the sets of losers, winners and indifferent agents in the manipulation at $R$ by $R^{\prime}$ :

$$
\begin{aligned}
& m \in \mathcal{W} \text { iff } u_{m}\left(Z_{m}^{\prime}\right)=\sum_{R_{m}} z_{m w}^{\prime}>u_{m}\left(Z_{m}\right) \\
& m \in \mathcal{I} \text { iff } u_{m}\left(Z_{m}^{\prime}\right)=u_{m}\left(Z_{m}\right) \\
& m \in \mathcal{L} \text { iff } u_{m}\left(Z_{m}^{\prime}\right)<u_{m}\left(Z_{m}\right)
\end{aligned}
$$


We assume $R_{m}=R_{m}^{\prime}$ for all $m \in \mathcal{L}$ (the losers cannot be part of the deviating coalition) and prove by induction $\mathcal{W}=\emptyset$. This establishes groupstrategyproofness because $R, R^{\prime}$ are arbitrary.

We write $\alpha_{k}, T_{k}, W_{k}$, and $\alpha_{l}^{\prime}, T_{l}^{\prime}, W_{l}^{\prime}$, for the sequences corresponding to $R$ and $R^{\prime}$ respectively. We prove by induction the property $\mathcal{P}(k)$ :

$$
T_{1, \ldots, k} \subseteq \mathcal{I} \cup \mathcal{L} ; z_{m w}^{\prime}=0 \text { for } w \in R\left(T_{1, \ldots, k}\right) \text { and } m \in(\mathcal{W} \cup \mathcal{I}) \cap T_{k+1, \ldots, K}
$$

We assume $\mathcal{P}(k-1)$ and prove $\mathcal{P}(k)$. In the case $k=1, \mathcal{P}(0)$ is void. We assume $k \leq K-1$, so that $u_{m}\left(Z_{m}\right)=\alpha_{k}$ for all $m \in T_{k}$. We set $T_{k}^{-}=T_{k} \cap \mathcal{L}$, $T_{k}^{+}=T_{k} \cap(\mathcal{W} \cup \mathcal{I})$, either of which can be empty. For any $m^{\prime} \in T_{k}^{-}, m \in T_{k}^{+}$, we have:

$$
u_{m^{\prime}}^{\prime}\left(Z_{m^{\prime}}^{\prime}\right)=u_{m^{\prime}}\left(Z_{m^{\prime}}^{\prime}\right)<u_{m^{\prime}}\left(Z_{m^{\prime}}\right)=\alpha_{k}=u_{m}\left(Z_{m}\right) \leq u_{m}\left(Z_{m}^{\prime}\right) \leq u_{m}^{\prime}\left(Z_{m}^{\prime}\right)
$$

where the first equality comes from $R_{m^{\prime}}=R_{m^{\prime}}^{\prime}$ and the last inequality holds because the support of $Z_{m}^{\prime}$ is contained in $R_{m}^{\prime}$. Therefore $m^{\prime}$ belongs to an "earlier" member of the partition $T_{l}^{\prime}$-a set with a smaller index $l$ - than $m$, implying that no object that $m^{\prime}$ likes (at $R_{m^{\prime}}=R_{m^{\prime}}^{\prime}$ ) is assigned to $m$ at $R^{\prime}: z_{m w}^{\prime}=0$ for $w \in R_{m^{\prime}}$. By the assumption $\mathcal{P}(k-1), Z^{\prime}$ does not give to $m$ any share of an object from $R\left(T_{1, \ldots, k-1}\right)$ either. Therefore:

$$
\left\{w \in R_{m} \text { and } z_{m w}^{\prime}>0\right\} \Rightarrow\left\{w \in R\left(T_{k}, W_{k-1}\right) \backslash R\left(T_{k}^{-}\right)\right\} \text {for all } m \in T_{k}^{+}
$$

Now we define an allocation matrix $Z^{*}$ restricted to $T_{k}, R\left(T_{k}, W_{k-1}\right)$, where $Z \rightarrow p(Z)$ simply deletes the coordinates outside $R\left(T_{k}, W_{k-1}\right)$ :

$$
\begin{aligned}
Z_{m^{\prime}}^{*} & =p\left(Z_{m^{\prime}}\right) \text { for } m^{\prime} \in T_{k}^{-} \\
Z_{m}^{*} & =p\left(Z_{m}^{\prime}\right) \text { for } m \in T_{k}^{+}
\end{aligned}
$$

By the definitions of $T_{k}$ and $W_{k-1}, u_{m^{\prime}}\left(Z_{m^{\prime}}^{*}\right)=\alpha_{k}$ for all $m^{\prime} \in T_{k}^{-}$, and by (21) $u_{m}\left(Z_{m}^{*}\right)=u_{m}\left(Z_{m}^{\prime}\right) \geq \alpha_{k}$ for all $m \in T_{k}^{+}$. Next the support of any $Z_{m^{\prime}}^{*}$ and that of any $Z_{m}^{*}$ are disjoint: see (21). Therefore $Z^{*}$ is feasible. By definition of $T_{k}$, all inequalities $u_{m}\left(Z_{m}^{*}\right) \geq \alpha_{k}$ must be equalities, which proves $T_{k}^{+} \subseteq \mathcal{I}$, and the first statement in $\mathcal{P}(k)$.

Moreover $Z^{*}$ must exhaust all objects of $R\left(T_{k}, W_{k-1}\right)$. Among these, those in $R\left(T_{k}, W_{k-1}\right) \backslash R\left(T_{k}^{-}\right)$are assigned in full by $Z^{\prime}$ to the agents of $T_{k}^{+}$: no fraction of those objects goes to anyone in $T_{k+1, \ldots, K}$. To complete the proof of $\mathcal{P}(k)$ we must check that the objects in $R\left(T_{k}^{-}\right)$are not assigned at all to any agent $n$ in $(\mathcal{W} \cup \mathcal{I}) \cap T_{k+1, \ldots, K}$ by $Z^{\prime}$. For such an agent $n$ we have: 


$$
u_{n}^{\prime}\left(Z_{n}^{\prime}\right) \geq u_{n}\left(Z_{n}^{\prime}\right) \geq u_{n}\left(Z_{n}\right) \geq \alpha_{k+1}
$$

therefore (20) implies $u_{n}^{\prime}\left(Z_{n}^{\prime}\right)>u_{m^{\prime}}^{\prime}\left(Z_{m^{\prime}}^{\prime}\right)$ for all $m^{\prime} \in T_{k}^{-}$.

Thus $m^{\prime}$ appears in the sequence $T_{l}^{\prime}$ earlier than $n$, implying that all objects he likes (at $R_{m^{\prime}}=R_{m^{\prime}}^{\prime}$ ) are allocated before $n$ is served, i.e., $n$ gets none of $R\left(T_{k}^{-}\right)$.

The proof of $\mathcal{P}(K-1)$ is now complete. If $\alpha_{K} \leq 1$, then $u_{m}\left(Z_{m}\right)=\alpha_{K}$ for $m \in T_{K}$ and the above argument shows $\mathcal{P}(K)$. If $\alpha_{K} \geq 1$, then $u_{m}\left(Z_{m}\right)=1$ implies $T_{K} \cap \mathcal{W}=\emptyset$. Thus $\mathcal{W}$ is empty in both cases.

\section{Theorem 3:}

The first statement is proven by means of the $4 \times 4$ example just before the theorem. To prove the second statement, fix an efficient welfarist solution $f$, and consider the same $4 \times 4$ example. We write $u=f(R)$ for the utility profile chosen by $f$. Without loss of generality we can assume $u_{m_{2}} \geq u_{m_{3}} \geq u_{m_{4}}$ and $v_{w_{2}} \geq v_{w_{3}} \geq v_{w_{4}}$; as $m_{1}$ and $w_{1}$ are overdemanded, $u_{m_{1}}=v_{w_{1}}=1$. This implies $u_{m_{3}}, v_{w_{3}} \leq \frac{1}{2}$ and $u_{m_{4}}, v_{w_{4}} \leq \frac{1}{3}$.

Consider the same manipulation $R M^{\prime}, R W^{\prime}$ by the coalition $S=\left\{m_{3}, m_{4}, w_{3}, w_{4}\right\}$. By efficiency, $f\left(R^{\prime}\right)=(1,1,1,1)$. Let $g$ be a solution projecting on $f$ and such that $g\left(R^{\prime}\right)$ is the allocation matrix:

$$
g\left(R^{\prime}\right)=\begin{array}{cccc}
0 & 0 & \frac{7}{12} & \frac{5}{12} \\
0 & 0 & \frac{5}{12} & \frac{7}{12} \\
\frac{7}{12} & \frac{5}{12} & 0 & 0 \\
\frac{5}{12} & \frac{7}{12} & 0 & 0
\end{array}
$$

Then $Z^{\prime}=g\left(R^{\prime}\right) \bullet R$ is:

$$
Z^{\prime}=\begin{array}{cccc}
0 & 0 & \frac{7}{12} & \frac{5}{12} \\
0 & 0 & 0 & 0 \\
\frac{7}{12} & 0 & 0 & 0 \\
\frac{5}{12} & 0 & 0 & 0
\end{array}
$$

and the (true) utility profile of $S$ after the misreport is $u_{m_{3}}^{\prime}=v_{w_{3}}^{\prime}=\frac{7}{12}>\frac{1}{2}$, $u_{m_{4}}^{\prime}=v_{w_{4}}^{\prime}=\frac{5}{12}>\frac{1}{3}$. Therefore $g$ is not weakly groupstrategyproof, and neither is $f$.

\section{Proofs for Section 7}

The straightforward proof of the Corollary to Theorem 1 is omitted for brevity.

Theorem 4

For the convenience of exposition, we regard assignment $\mu$ as a function from $M$ to $W$. Thus, $\mu(m)=w$ means that an agent $m$ is assigned to an object $w$. 
1) Random Priority Solution

a) Fair Share

The probability that agent $m$ comes up among the first $\left|R_{m}\right|$ agents in the random ordering of $\succ$ is $\frac{\left|R_{m}\right|}{|M|}$. When this happens, at least one object in $R_{m}$ is still available when agent $m$ gets to choose. Fair share follows.

b) No Envy

We fix two agents 1,2 and an arbitrary ordering $\succ$ of $M$ where 1 precedes 2. We write $\succ^{*}$ for the ordering where 1 and 2 are permuted, everything else unchanged. We select a $\succ$ priority assignment $\mu$, and $\mu^{*}$ for $\succ^{*}$. We check that agent 1 does not envy agent 2 in the assignment matrix $Z=\frac{1}{2}\left(\mu+\mu^{*}\right)$. This establishes No Envy because every random priority allocation matrix $Z$ obtains by convex combinations of such matrices, and the inequality (13) is preserved by such combinations.

Let $T$ be the coalition preceding 1 and 2 in $\succ$ and $\succ^{*}$, and $S$ that in between 1 and 2 in both $\succ$ and $\succ^{*}$. Assume, without loss of generality, that 1 precedes 2 in $\succ$ and follows him in $\succ^{*}$. If $u_{1}(\mu)=0$, i.e., $1 \notin S(\succ)$ (see Lemma 4 ) then agent 1 does not like any object that 2 may get under $\mu$, otherwise we could have given such an object to 1 without affecting the utilities of $T$, contradiction. Similarly, 1 does not like any object 2 may get under $\mu^{*}$, so 1 does not envy 2 at $Z$ if $u_{1}(\mu)=0$. If $u_{1}(\mu)=1$, the only way 1 could envy 2 at $Z$ is if:

$$
\mu(1)=a, \mu(2)=b ; \mu^{*}(2)=c, \mu^{*}(1)=\emptyset ; 1 \text { likes } a, b, c
$$

where $a \neq b$ but object $c$ could be equal to $a$ or $b$.

Let $w, w^{*}$ be the $S$-utility profiles at $\succ$ and $\succ^{*}$ respectively. Write lex for the $\succ$ lexicographic ordering of $[0,1]^{S}$. Let $\mu^{\prime}$ be equal to $\mu^{*}$ on $T$ and $S$, and $\mu^{\prime}(1)=c$ : it gives the same utility as $\mu$ to $T$ and 1 , and the same as $\mu^{*}$ to $S$, therefore $w \operatorname{lex} w^{*}$. Now compare the full utility profiles $u$ at $\mu$ and $u^{*}$ at $\mu^{*}$ : they coincide for $T$ and 2 , on $S u$ is $\succ$ lexicographically superior or equal to $u^{*}$, and $u_{1}>u_{1}^{*}$. This contradicts the definition of $u^{*}$.

c) Population and resource monotonicity. The Shapley value of a concave (submodular) cooperative game is population monotonic (Sprumont [1990]), and the random priority utility profile in Definition 1 is the Shapley value of the game $T \rightarrow e(T, W, \widetilde{R})$. The supermodularity of $e(T, B, R)$ with respect to $T, B$ (Lemma 3 ) and Lemma 4 imply that a fixed priority mechanism is resource monotonic; this property is stable by convex combinations.

2) Egalitarian solution

a) Fair Share. If $m \in M^{a}$, there is nothing to prove. Pick $m \in M^{d}$, and $m \in T_{l}$. Recall that the sets $R\left(T_{k}, W_{k-1}\right), k=1, \ldots, l$, form a partition of $R\left(T_{1, \ldots, l}\right)$ and:

$$
\frac{r\left(T_{k}, W_{k-1}\right)}{\left|T_{k}\right|}<\frac{r\left(T_{l}, W_{l-1}\right)}{\left|T_{l}\right|}=u_{m} \text { for } k=1, \ldots, l . \text { Hence } \frac{\left|R\left(T_{1, \ldots, l}\right)\right|}{\left|T_{1, \ldots, l}\right|} \leq u_{m}
$$


Inequality (12) follows because $R\left(T_{1, \ldots, l}\right)$ contains $R_{m}$.

b) No Envy. An equal income competitive allocation meets No Envy: this well-known fact applies here to any "equal income competitive" allocation matrix $Z$, as defined in the Corollary.

c) Population monotonicity. Lemma 5.6 in Dutta [1990] shows that the egalitarian solution is population monotonic. As shown in the proof of Theorem 1 , the profile $u^{e}$ is the egalitarian solution of the concave game $T \rightarrow e(T, W, \widetilde{R})$.

d) Resource monotonicity. Fix a problem $(M, W, R)$ and an object $w \in W$. The utility profiles and sequences (9) for the initial problem and the reduced problem $(M, W \backslash w, \widetilde{R})$ are denoted $u, \alpha_{k}, T_{k}, \ldots$ and $u^{\prime}, \alpha_{k}^{\prime}, T_{k}^{\prime} \ldots$ respectively. Suppose, to the contrary, that $u^{\prime} \leq u$ fails. Then there is an agent $m$ and an index $k$ such that:

$$
m \in T_{k} ; u_{m}^{\prime}>u_{m}=\alpha_{k} ; u_{m^{\prime}}^{\prime} \leq u_{m^{\prime}} \text { for all } m^{\prime} \in T_{1}, \ldots, k-1
$$

Let $m \in T j$ in the algorithm of the reduced problem. We claim $T_{1, \ldots, k-1} \subseteq$ $T_{1, \ldots, j-1}^{\prime}$. Indeed for all $m^{\prime} \in T_{1, \ldots, k-1}$ we have $u_{m^{\prime}}^{\prime} \leq u_{m^{\prime}}<u_{m}<u_{m}^{\prime} \leq \alpha_{j}^{\prime}$ therefore $m^{\prime} \in T_{1, \ldots, m-1}^{\prime}$. Notice that $u_{m}^{\prime}<\alpha_{j}^{\prime}$ is possible if $u_{m}^{\prime}=1$, but $u_{m}=\alpha_{k}$ as $u_{m}<1$.

The set $V=T_{k} \backslash T_{1, \ldots, j-1}^{\prime}$ is non-empty: it contains agent $m$. Set $V^{\prime}=$ $T_{k} \cap T_{1, \ldots, j-1}^{\prime}$ (possibly empty). We show next:

$$
\frac{r\left(V, W_{k-1} \backslash R\left(V^{\prime}\right)\right)}{|V|} \leq \alpha_{k}
$$

where $R, r$ refer to the problem $(M, W, R)$. If $V^{\prime}=\emptyset, V=T_{k}$ we have an equality. If $V^{\prime} \neq \emptyset$ we have $\frac{r\left(V^{\prime}, W_{k-1}\right)}{\left|V^{\prime}\right|} \geq \alpha_{k}$, so if (23) is false we get a contradiction:

$$
\alpha_{k}<\frac{r\left(V^{\prime}, W_{k-1}\right)+r\left(V, W_{k-1} \backslash R\left(V^{\prime}\right)\right)}{|V|+\left|V^{\prime}\right|}=\frac{r\left(T, W_{k-1}\right)}{\left|T_{k}\right|}
$$

Now we check $W_{j-1}^{\prime} \subseteq W_{k-1} \backslash R\left(V^{\prime}\right)$, where $W_{j-1}^{\prime}$ refers to the reduced problem $(M, W \backslash w, \widetilde{R})$ :

$$
\begin{aligned}
W_{j-1}^{\prime} & =(W \backslash w) \backslash R\left(T_{1, \ldots, j-1}^{\prime}\right) \subseteq W \backslash R\left(T_{1, \ldots, j-1}^{\prime}\right) \\
& \subseteq W \backslash R\left(T_{1, \ldots, k-1} \cup V^{\prime}\right)=W_{k-1} \backslash R\left(V^{\prime}\right)
\end{aligned}
$$

We get finally the desired contradiction:

$$
u_{m}^{\prime} \leq \alpha_{j}^{\prime} \leq \frac{r\left(V, W_{j-1}^{\prime}\right)}{|V|} \leq \frac{r\left(V, W_{k-1} \backslash R\left(V^{\prime}\right)\right)}{|V|} \leq \alpha_{k}=u_{m}
$$




\section{3) Uniform solution}

a) Fair Share: Fix $(M, W, R)$ and an agent $m \in M^{d}$. We restrict attention to the problem $\left(M^{d}, W^{o}, \widetilde{R}\right)$, that we write $(M, W, R)$ for simplicity.

Denote $E=E(M, W, R), E_{m}=E_{m}(M, W, R)$ and $E_{-m}=E \backslash E_{m}$, all nonempty. Define a bilateral graph between $E_{-m}$ and $E_{m}$ as follows: $S \in E_{-m}$ and $T \in E_{m}$ are matched if $T \backslash m \subseteq S$.

Note that each $T \in E_{m}$ is matched with at most $|M|-|W|$ coalitions $S$ in $E_{-m}$. Indeed, all coalitions in $E$ are of the same size $|W|$, so $T \backslash m \subseteq S$ implies $|S \backslash T|=1$, this unique agent being from $M \backslash T$.

We claim that each $S \in E_{-m}$ is matched with at least $\left|R_{m}\right|$ coalitions $T$ in $E_{m}$. For if $\mu$ assigns $S$, each object in $R_{m}$ must be assigned (by (6)); thus for each $w \in R_{m}$, we create an efficient assignment by giving $w$ to $m$ in lieu of $\mu^{-1}(w)$, and the corresponding coalitions in $E_{m}$ are all distinct. This proves the claim.

Let $k$ be the number of pairs $(S, T)$ that are matched. Denoting $p=|M|-$ $|W|$ we have shown that $k \leq p \cdot\left|E_{m}\right|$ and $k \geq\left|R_{m}\right| \cdot\left|E_{-m}\right|$. Hence:

$$
u_{m}=\frac{\left|E_{m}\right|}{\left|E_{m}\right|+\left|E_{-m}\right|} \geq \frac{\left|R_{m}\right|}{p+\left|R_{m}\right|}
$$

The desired inequality (12) follows at once.

b) No Envy. Fix $(M, W, R)$ and two agents $m, m^{\prime}$. If an agent is in $M^{a}$ she is neither envious nor envied (Lemma 1) therefore we can assume $m, m^{\prime} \in M^{d}$ and restrict attention to $\left(M^{d}, W^{o}, \widetilde{R}\right)$ as in the proof of Fair Share. Let $E_{m^{\prime}}^{*}$ be the subset of $E_{m^{\prime}}$ made of those $S$ such that in at least one assignment where everyone in $S$ is assigned, $m^{\prime}$ receives an object in $R_{m}$. Then the coalition $\delta(S)$ given below is in $E_{m}$ for all $S$ in $E_{m^{\prime}}^{*}$ :

$$
\begin{aligned}
\delta(S) & =S \quad \text { if } S \in E_{m^{\prime}}^{*} \cap E_{m} \\
& =\left(S \backslash m^{\prime}\right) \cup m \text { if } S \in E_{m^{\prime}}^{*} \cap E_{-m}
\end{aligned}
$$

Clearly $\delta$ is one-to-one, thus $\left|E_{m^{\prime}}^{*}\right| \leq\left|E_{m}\right|$. Finally, in any allocation matrix $Z$ implementing the uniform solution, $u_{m}\left(z_{m^{\prime}}\right) \leq \frac{\left|E_{m^{\prime}}^{*}\right|}{|E|}$ implying $u_{m}\left(z_{m^{\prime}}\right) \leq \frac{\left|E_{m}\right|}{|E|}$ as desired. 